\title{
GALAXY HALO TRUNCATION AND GIANT ARC SURFACE BRIGHTNESS RECONSTRUCTION IN THE CLUSTER MACSJ1206.2-0847
}

\author{
Thomas Eichner ${ }^{1,2}$, Stella Seitz ${ }^{1,2}$, Sherry H. Suyu ${ }^{3,4,5}$, Aleksi Halkola ${ }^{6}$, Keitchi Umetsu ${ }^{5}$, Adi Zitrin ${ }^{7}$, Dan CoE $^{8}$, \\ Anna Monna ${ }^{1,2}$, Piero Rosati ${ }^{9}$, Claudio Grillo ${ }^{10}$, Italo Balestra $^{2}$, Marc Postman $^{8}$, Anton Koekemoer ${ }^{8}$, Wei Zheng ${ }^{11}$, \\ Ole Høst $^{10}$, Doron Lemze ${ }^{11}$, Tom Broadhurst ${ }^{12}$, Leonidas Moustakas ${ }^{13}$, Larry Bradley ${ }^{8}$, Alberto Molino ${ }^{14}$, \\ Mario Nonino $^{15}$, Amata Mercurio ${ }^{16}$, Marco ScodegGio ${ }^{17}$, Matthias Bartelmann ${ }^{7}$, Narciso Benitez ${ }^{14}$, \\ Rychard Bouwens $^{18}$, Megan Donahue ${ }^{19}$, Leopoldo Infante ${ }^{20}$, Stephanie Jouvel ${ }^{21,22}$, Daniel Kelson ${ }^{23}$, Ofer Lahav ${ }^{21}$, \\ Elinor Medezinski ${ }^{11}$, Peter Melchior $^{24}$, Julian Merten $^{13}$, and Adam Riess $^{8,11}$ \\ ${ }^{1}$ Universitäts-Sternwarte München, Scheinerstr. 1, D-81679 München, Germany \\ 2 Max-Planck-Institut für Extraterrestrische Physik, Giessenbachstraße, D-85748 Garching, Germany \\ ${ }^{3}$ Department of Physics, University of California, Santa Barbara, CA 93106, USA \\ ${ }^{4}$ Kavli Institute for Particle Astrophysics and Cosmology, Stanford University, 452 Lomita Mall, Stanford, CA 94035, USA \\ 5 Institute of Astronomy and Astrophysics, Academia Sinica, P.O. Box 23-141, Taipei 10617, Taiwan \\ ${ }^{6}$ Institute of Medical Engineering, University of Lübeck, Ratzeburger Allee 16023562 Lübeck, Germany \\ ${ }^{7}$ Institut für Theoretische Astrophysik, ZAH, Albert-Ueberle-Straße 2, D-69120 Heidelberg, Germany \\ ${ }^{8}$ Space Telescope Science Institute, 3700 San Martin Drive, Baltimore, MD 21208, USA \\ ${ }^{9}$ ESO-European Southern Observatory, D-85748 Garching bei München, Germany \\ ${ }^{10}$ Dark Cosmology Centre, Niels Bohr Institute, University of Copenhagen, Juliane Maries Vej 30, DK-2100 Copenhagen, Denmark \\ ${ }^{11}$ Department of Physics and Astronomy, The Johns Hopkins University, 3400 North Charles Street, Baltimore, MD 21218, USA \\ 12 Department of Theoretical Physics, University of the Basque Country, P.O. Box 644, E-48080 Bilbao, Spain \\ 13 Jet Propulsion Laboratory, California Institute of Technology, MS 169-327, Pasadena, CA 91109, USA \\ ${ }^{14}$ Instituto de Astrofísica de Andalucía (CSIC), C/Camino Bajo de Huétor 24, Granada E-18008, Spain \\ 15 INAF-Osservatorio Astronomico di Trieste, via G.B. Tiepolo 11, I-40131 Trieste, Italy \\ ${ }^{16}$ INAF-Osservatorio Astronomico di Capodimonte, via Moiariello 16, I-80131 Napoli, Italy \\ ${ }^{17}$ INAF-IASF Milano, Via Bassini 15, I-20133 Milano, Italy \\ ${ }^{18}$ Leiden Observatory, Leiden University, P.O. Box 9513, 2300 RA Leiden, The Netherlands \\ ${ }^{19}$ Department of Physics and Astronomy, Michigan State University, East Lansing, MI 48824, USA \\ ${ }^{20}$ Departamento de Astronoía y Astrofísica, Pontificia Universidad Católica de Chile, V. Mackenna 4860, Santiago 22, Chile \\ ${ }^{21}$ Department of Physics and Astronomy, University College London, Gower Street, London WCIE 6 BT, UK \\ 22 Institut de Cincies de l'Espai (IEEC-CSIC), Bellaterra (Barcelona), Spain \\ 23 Observatories of the Carnegie Institution of Washington, Pasadena, CA 91101, USA \\ ${ }^{24}$ Center for Cosmology and Astro-Particle Physics, and Department of Physics, The Ohio State University, \\ 191 W. Woodruff Ave., Columbus, OH 43210, USA \\ Received 2012 October 12; accepted 2013 June 19; published 2013 August 23
}

\begin{abstract}
In this work, we analyze the mass distribution of MACSJ1206.2-0847, particularly focusing on the halo properties of its cluster members. The cluster appears relaxed in its X-ray emission, but has a significant amount of intracluster light that is not centrally concentrated, suggesting that galaxy-scale interactions are still ongoing despite the overall relaxed state. The cluster lenses 12 background galaxies into multiple images and one galaxy at $z=1.033$ into a giant arc and its counterimage. The multiple image positions and the surface brightness (SFB) distribution of the arc, which is bent around several cluster members, are sensitive to the cluster galaxy halo properties. We model the cluster mass distribution with a Navarro-Frenk-White profile and the galaxy halos with two parameters for the mass normalization and the extent of a reference halo assuming scalings with their observed near-infrared light. We match the multiple image positions at an rms level of 0.85 and can reconstruct the SFB distribution of the arc in several filters to a remarkable accuracy based on this cluster model. The length scale where the enclosed galaxy halo mass is best constrained is about 5 effective radii-a scale in between those accessible to dynamical and field strong-lensing mass estimates on the one hand and galaxy-galaxy weak-lensing results on the other hand. The velocity dispersion and halo size of a galaxy with $m_{160 \mathrm{~W}, \mathrm{AB}}=19.2$ and $M_{\mathrm{B} \text {, Vega }}=-20.7$ are $\sigma=150 \mathrm{~km} \mathrm{~s}$ and $r \approx 26 \pm 6 \mathrm{kpc}$, respectively, indicating that the halos of the cluster galaxies are tidally stripped. We also reconstruct the unlensed source, which is smaller by a factor of $\sim 5.8$ in area, demonstrating the increase in morphological information due to lensing. We conclude that this galaxy likely has star-forming spiral arms with a red (older) central component.
\end{abstract}

Key words: galaxies: clusters: individual (MACSJ1206.2-0847) - galaxies: elliptical and lenticular, cD - galaxies: halos - galaxies: interactions - gravitational lensing: strong

Online-only material: color figures

\section{INTRODUCTION}

For elliptical galaxies, their half-light radii, central velocity dispersions, and surface brightnesses (SFBs) within their halflight radii form a fundamental plane (FP; Bender et al. 1992). This FP relation is very similar for field and cluster galaxies at the same redshift (Andreon 1996; Saglia et al. 2010). The redshift evolution of the elliptical galaxies' mass-to-light ratios is independent of the cluster velocity dispersion; it is compatible with passive evolution of the stellar population (Bender et al. 1998; van Dokkum \& van der Marel 2007; Saglia et al. 2010) and slightly stronger for field galaxies. The effective radii and 
velocity dispersions of elliptical galaxies evolve with time, but do not depend significantly on galaxy environment.

Thomas et al. $(2005,2009)$ studied elliptical dark matter halos with stellar dynamics and showed that (1) the stars of elliptical galaxies formed at high redshift $(z=3-5),(2)$ the dark matter halos of (Coma) elliptical galaxies formed earlier than those of spiral galaxies of same brightness and environment, and (3) the halos of elliptical galaxies mostly formed at least as early as their stars (see Figure 13 of Wegner et al. 2012).

In general, however, galaxy environment plays a major role in the formation of galaxies and the transformation of galaxy types according to the morphology-density relation of Dressler (1980). Environment furthermore affects the evolution of galaxies with redshift (Dressler et al. 1997). Dressler et al. (1997, p. 577) concluded that "the formation of elliptical galaxies predates the formation of rich clusters, and occurs instead in the loose-group phase or even earlier." Wilman \& Erwin (2012) confirmed this picture in a quantitative way: according to their interpretation, elliptical galaxies are centrals or they are satellites that had been centrals in halos before they were accreted. Taken together, this result implies that the central stellar dynamics and the stellar population content of elliptical galaxies depend, to a minor level, on their present-day environment. Elliptical galaxies stay elliptical galaxies when larger-scale halos like those of groups and clusters form, but depending on their evolution into central or satellite galaxies their dark matter halos undergo growth or stripping.

The stripping of dark matter halos embedded in group and cluster halos by tidal fields is theoretically expected (Merritt 1983, 1984) and increases in strength in denser environments. Stripping has also been studied in $N$-body dark matter simulations (Ghigna et al. 1998; Limousin et al. 2009). Gao et al. (2004b) have shown that, on average, $90 \%$ of the mass associated with halos accreted at $z=1$ is removed from the accreted halos and contributes to the smooth host halo at $z=0$. The highest mass accreted halos reach the center more quickly, due to dynamical friction, and thus become stripped most quickly. Diemand et al. (2007) have shown that subhalo mass is removed starting from the outside, in agreement with the observations that any changes of the FP relation with environment can be explained by slight age differences of the stellar populations, i.e., that the structural parameters of elliptical galaxies do not change during the build up of groups and clusters. Warnick et al. (2008) have shown that, on average, surviving subhalos lose about $30 \%$ of their mass per orbit in group and cluster halos (this excludes tidally disrupted halos), whereas halos with radial orbits may lose $80 \%$ or even more of their mass per orbit. Figure 4 in Warnick et al. (2008) illustrates the subhalo mass loss sorted as a function of subhalo distance to the halo center, for different central halo masses. Within $10 \%$ of the virial radius, the majority of subhalos have lost more than $50 \%$ of their original mass. Limousin et al. (2009) studied galaxy dark matter halo truncation in high-density environments with hydrodynamical $N$-body simulations. They predict the half-light radii of galaxies in a Coma- and Virgo-like cluster as a function of three-dimensional (3D) and two-dimensional (2D) projected separation to the cluster center, finding a measurable effect in both, at a level stronger than that of Ghigna et al. (1998). According to their work, the total mass of galaxy halos is a few times larger than the stellar mass in the center and up to about 200 (50) times larger in the outskirts of the cluster at $z=0.7(z=0)$.

Galaxy halo stripping in clusters has been measured with planetary nebula kinematics in local galaxies (Ventimiglia et al. 2011, and references therein). Pu et al. (2010) analyzed the stellar kinematics of massive local elliptical galaxies and measured halo sizes of orders of $60 \mathrm{kpc}$ based on the Mgb absorption line strength versus escape velocity relation. These analysis methods for individual galaxy halos do not yet work for larger samples and even larger distances.

Galaxy halo sizes can also be measured with weak galaxy-galaxy lensing for field galaxies (Schneider \& Rix 1997; Hoekstra et al. 2004) and also for cluster galaxies using statistical methods and large samples. In clusters, the effect is stronger per galaxy since the signal is boosted by the matter of the cluster itself (Geiger \& Schneider 1999), but this also imposes a degeneracy in measuring the galaxy halos (Geiger \& Schneider 1999). Nevertheless, halo truncation has been measured with weak galaxy-galaxy lensing (Narayan 1998; Geiger \& Schneider 1999; Natarajan et al. 2002a, 2002b; Limousin et al. 2007a) and truncations in half-mass radii by a factor of four as compared to field galaxies have been reported.

Halkola et al. (2007) worked out a different idea: using strong gravitational lensing, they described the mass distribution in the massive strong-lensing cluster A1689 with a smooth dark matter component and a smaller-scale component traced by the cluster galaxies. The combined "granular" mass distribution maps multiply imaged galaxies differently than the best-fitting pure smooth cluster component. Making use of the FP and Faber-Jackson (FJ) scaling relations for cluster galaxies, the properties of a reference halo could be measured. This method finds the statistically best-fitting reference galaxy halo mass distribution that best reproduces the astrometry of the multiplyimaged sources. It relies on a very precise global mass model (Broadhurst et al. 2005; Halkola et al. 2006; Limousin et al. 2007b, see also Diego et al. 2005; Coe et al. 2010) constrained by a huge number of multiple images (in this case, 32 background galaxies mapped into 107 images) spread over the Einstein radii corresponding to the various source redshifts.

Studying the impact of substructure in the lens with multiple image positions does not make use of the full information, since this method simply employs the different deflection angles between multiply imaged sources neglecting higher order or local derivatives of the deflection angle. This can be done when mapping the full SFB distribution of the images and adjusting the model such that for every image system of a reproduced source, the SFBs match the observations. Colley et al. (1996) were the first to measure the unlensed SFB distribution of the five image system in $\mathrm{Cl0024}$ and utilizing this to constrain the mass distribution of the cluster. Seitz et al. (1998) analyzed the lensing effect of the cluster MS1512 using several multiply-imaged systems and obtained the SFB distribution of the highly magnified galaxy cB58 to a unprecedented spatial resolution. In this analysis, it was important to account for the mass distribution of a galaxy perturbing the cB58 arc such that it was bent away from the cluster center-although measuring galaxy halos was not the aim of this work.

Later on, Suyu \& Halkola (2010) analyzed the SFB distribution of a source multiply imaged by a galaxy with a satellite as a perturber. These authors could indeed measure the satellite halo size in this way, showing that the sensitivity of this method can be extended to (still massive) satellites in favorable lensing systems. On the cluster lens scale, Donnarumma et al. (2011) used a method similar to that of Halkola et al. (2007) to constrain halo sizes in A611. In this case, one of the sources is mapped into a giant arc system, of which several corresponding SFB knots are 
used for lens modeling. This technique partially makes use of the SFB distribution of the arc in this cluster.

In this work, we study galaxy halo truncation in the cluster MACSJ1206.2-0847; it is an ideal target for several reasons: MACSJ1206.2 is a massive cluster at redshift $z=0.439$ (for a summary of its properties and lensing, X-ray, and Sunyaev-Zeldovich effect results, see Umetsu et al. 2012 and Zitrin et al. 2012b). This cluster still shows signs of its recent assembly, since there is a "trail" of intracluster light along its major axis (in mass and light), indicating previous tidal stripping down to the core of galaxies or tidal disruption of galaxies. On the other hand, its central galaxy is almost at rest relative to the center of mass (as obtained from cluster members' velocities); see Biviano et al. (2013). Furthermore, this cluster appears relaxed based on its X-ray contours (Ebeling et al. 2009; Umetsu et al. 2012). This means that the cluster members orbited each other for at least a significant fraction of the crossing time, were exposed to the dense cluster environment, and had the necessary (and short) time to become tidally stripped. Due to its deep multiband Hubble Space Telescope (HST) photometry, this cluster is observed to have many multiple image systems (Zitrin et al. 2012b) and furthermore has a giant arc, which is bent around several cluster members, making the light deflection of the galaxy halos visible to the eye. Using the SFB distribution of the arcs and the multiple image positions, this cluster thus offers the opportunity to provide very strong constraints on halo sizes.

This paper is organized as follows. In Section 2, we give an overview of the data used, in Section 3 we present the models for the mass distribution of the cluster and the halos traced by cluster galaxies, and in Section 4 we introduce the scaling relations connecting galaxy luminosity and dark matter halo properties. In Section 5, we obtain a strong-lensing model using only point-source constraints from multiple images and the giant arc. Section 6 then also includes the full SFB distribution of the arc and its counterimage in the analysis. In Section 7, we discuss our results concerning the scaling of cluster galaxies' luminosities with their velocity dispersions and halo sizes and the properties of the unlensed source of the arc's counterimage. Section 8 gives a summary of the work and adds conclusions. We use a $W M A P 7^{25}$ (Komatsu et al. 2011) cosmology throughout the paper. This cosmology gives a scale of $5.662 \mathrm{kpc} \mathrm{arcsec}^{-1}$ at the redshift of the cluster, $z=0.439$. Einstein radii, convergence, and shear values are given in units of the ratio of the angular diameter distances from the lens to the source $\left(D_{\mathrm{ds}}\right)$ and the observer to the source $\left(D_{\mathrm{s}}\right), D_{\mathrm{ds}} D_{\mathrm{s}}^{-1}$, if not otherwise stated. Angles are measured in degrees $\mathrm{N}$ of $-\mathrm{E}$.

\section{DATA}

The data used in this work are described in Postman et al. (2012), Zitrin et al. (2012b), and Ebeling et al. (2009). All raw and reduced HST imaging data taken by CLASH are public. We obtain positions and shapes of cluster galaxies with Sextractor (Bertin \& Arnouts 1996) from the F606W filter data. The F435W, the F606W, and the F814W filter data are used to extract the SFB distribution of the arc and its counterimage for the lens modeling. We need an rms-noise estimate for each pixel of the giant gravitational arc and its counterimage for the SFB reconstruction. We obtain the prereduced, publicly available $F L T$ images for the F435W, F606W, and F814W filters, respectively. The pre-reduction, done by calacs, includes overscan and bias correction as well as flat-

$25 H_{0}=71 \mathrm{~km} \mathrm{~s}^{-1} \mathrm{Mpc}^{-1}, \Omega_{\mathrm{M}}=0.267$, and $\Omega_{\Lambda}=0.734$. fielding of the single images. Afterward, Multidrizzle was used for the alignment, background subtraction, cosmic-ray rejection, and weighted co-addition of the individual frames and the rms-noise estimate. The weighting scheme used is the ERR scheme, where the weighting is done by the inverse variance of each pixel. From this inverse variance, we calculate the rmsnoise estimate for each pixel. For these frames, we choose a pixel scale of 0.05 resembling the natural pixel scale of the Advanced Camera for Surveys (ACS). We verify that the corresponding stellar positions in the different filters are accurate to $\approx 0.5$ pixels.

\section{MODELING THE CLUSTER AND ITS GALAXY COMPONENT}

Since we want to measure the parameter values of halo truncation, we use parametric lens models. The main cluster component is modeled by a Navarro-Frenk-White (NFW; Navarro et al. 1997) halo. Its lensing properties are described in Wright \& Brainerd (2000) and Golse \& Kneib (2002):

$$
\begin{aligned}
\Sigma(X)=2 r_{\mathrm{s}} \delta_{\mathrm{c}} \rho_{\mathrm{c}} \\
\\
\quad \times \begin{cases}\frac{1}{X^{2}-1}\left[1-\frac{2}{\sqrt{1-X^{2}}} \operatorname{arctanh} \sqrt{\frac{1-X}{1+X}}\right] & X<1 \\
\frac{1}{3} & X=1 \\
\frac{1}{X^{2}-1}\left[1-\frac{2}{\sqrt{X^{2}-1}} \arctan \sqrt{\frac{X-1}{1+X}}\right] & X>1 .\end{cases}
\end{aligned}
$$

Here, $r_{\mathrm{s}}, \delta_{\mathrm{c}}$, and $\rho_{\mathrm{c}}$ are the scale radius, the characteristic overdensity of the halo, and the critical density of the universe for closure at the redshift of the halo, respectively. For the spherical case, $X=R / r_{\mathrm{s}}$ denotes the dimensionless distance in the image plane. Following Golse \& Kneib (2002) and Halkola et al. (2006), we introduce elliptical isopotential contours by introducing the axis ratio $q=b a^{-1}$ with major and minor axes $a$ and $b$, respectively. $X=\sqrt{x_{1}^{2} / q+x_{2}^{2} q}$ then denotes the nonspherical extension of the spherical case above, with $x_{1}$ and $x_{2}$ being the Cartesian coordinates in the major axis coordinate system. In the following, we will only consider the elliptical case, calling that the NFW profile.

We model the cluster galaxies as Brainerd et al. (1996) with their so-called BBS: the density profile is an isothermal sphere with a "velocity dispersion" $\sigma$ and a truncation radius $r_{t}$ :

$$
\rho(r)=\frac{\sigma^{2}}{2 \pi \mathrm{G} r^{2}} \frac{r_{\mathrm{t}}^{2}}{r^{2}+r_{\mathrm{t}}^{2}} .
$$

The projected surface mass density is

$$
\Sigma(R)=\frac{\sigma^{2}}{2 G R}\left[1-\left(1+\frac{r_{\mathrm{t}}^{2}}{R^{2}}\right)^{-0.5}\right] .
$$

This gives an enclosed mass within a cylinder of radius $R$ of

$$
M(<R)=\frac{\pi \sigma^{2}}{G}\left[R+r_{\mathrm{t}}-\sqrt{R^{2}+r_{\mathrm{t}}^{2}}\right],
$$

and a total mass of

$$
M_{\mathrm{tot}}=\frac{\pi \sigma^{2} r_{\mathrm{t}}}{G}
$$

where $G$ is the gravitational constant and $R$ is the $2 \mathrm{D}$ radius. For the exact lensing properties, see Brainerd et al. (1996). Following Halkola et al. (2006), ellipticity is again introduced 
in the potential in the same way as in the NFW case. The truncation radius $r_{\mathrm{t}}$ marks the transition region from a density slope $\rho \sim r^{-2}$ to a slope of $\rho \sim r^{-4}$. At $r_{\mathrm{t}}$, the projected density is half the value of the singular isothermal sphere model with the same $\sigma$. For the 3D density, the truncation radius is equal to the half-mass radius of the profile; see Elíasdóttir et al. (2007) and Limousin et al. (2009). For the 2D projected density, the 2D half-mass radius is smaller, $r_{1 / 2,2 D}=0.75 r_{\mathrm{t}}$.

\section{GALAXY SCALING RELATIONS}

We are not able to precisely constrain galaxy halo sizes for individual cluster members in this cluster. Therefore, we use scaling relations between the different galaxy halos, based on the luminosity of the individual galaxies, to estimate an average truncation for all halos. As in Halkola et al. $(2006,2007)$ and Limousin et al. (2007a), we make use of the FJ (previously defined) relation connecting the luminosity $(L)$ of early-type galaxies with their central stellar velocity dispersion $\sigma_{\text {star }}$ and halo velocity dispersion ${ }^{26} \sigma$ with reference values $\sigma^{\star}$ and $L^{\star}$ with power law exponent $\delta$ :

$$
\sigma=\sigma^{\star}\left(\frac{L}{L^{\star}}\right)^{\delta}
$$

We further assume that the truncation radius scales with luminosity as (Hoekstra et al. 2003; Halkola et al. 2006, 2007; Limousin et al. 2007a):

$$
r_{\mathrm{t}}=r_{\mathrm{t}}^{\star}\left(\frac{L}{L^{\star}}\right)^{\alpha}=r_{\mathrm{t}}^{\star}\left(\frac{\sigma}{\sigma^{\star}}\right)^{\alpha / \delta} .
$$

Here, $\sigma^{\star}$ and $r_{\mathrm{t}}^{\star}$ are the parameter values for a galaxy halo with a reference luminosity $L^{\star}$. In order to specify the scaling relations, we need to find appropriate values for $\alpha$ and $\alpha / \delta$. The values for the FJ slope $\delta$ quoted in literature depend on the wavelength range used for the luminosity measurement and on the considered magnitude range (Nigoche-Netro et al. 2011; Focardi \& Malavasi 2012). For the $B$-band relation, we will in the following consider slopes between $\delta=0.3$ (Ziegler $\&$ Bender 1997) and $\delta=0.25$ (Fritz et al. 2009; Kormendy \& Bender 2013; Focardi \& Malavasi 2012). Furthermore, Bernardi et al. (2003) find a value of $\delta=0.25$ for elliptical galaxies in each of the Sloan Digital Sky Survey (SDSS) $g^{*} r^{*} i^{*} z^{*}$ bands. However, there are indications for an increase in $\delta$ for fainter elliptical galaxies (see, e.g., Matković \& Guzmán 2005, and references therein). We therefore assume $\delta$ to be equal to 0.3 for our analysis. This value has also been found by Rusin et al. (2003) from gravitational lensing of field elliptical galaxies. The exact choice for $\delta$ is not relevant for our work, since we are not able to distinguish a scaling relation with a slope of, e.g., $\delta=0.27$ from one with a slope of 0.3 .

To limit the reasonable range for the truncation scaling $\alpha$, we consider the mass-to-light ratio of galaxies. The total mass-to-light ratio is usually described by a power law as well:

$$
\frac{M_{\mathrm{tot}}}{L} \propto L^{\epsilon} \propto \sigma^{\epsilon / \delta} .
$$

Using $M_{\mathrm{tot}} \propto \sigma^{2} r_{\mathrm{t}}$ (Equation (5)) with Equations (6) and (7), we obtain for the same mass-to-light ratio

$$
\frac{M_{\mathrm{tot}}}{L} \propto \sigma^{2+\alpha / \delta-1 / \delta},
$$

\begin{tabular}{|c|c|c|c|c|c|c|c|}
\hline \multicolumn{4}{|c|}{ Field Galaxies } & \multicolumn{4}{|c|}{ Stripped Galaxies } \\
\hline$\delta$ & $\epsilon$ & $\frac{\alpha}{\delta}$ & $\alpha$ & $\delta$ & $\frac{\alpha}{\delta}$ & $\alpha$ & $\epsilon_{\text {stripped }}$ \\
\hline 0.3 & 0.2 & 2 & 0.6 & 0.30 & 1 & 0.30 & -0.10 \\
\hline 0.25 & 0.0 & 2 & 0.5 & 0.25 & 1 & 0.25 & -0.25 \\
\hline 0.25 & 0.2 & 2.8 & 0.7 & 0.233 & 1 & 0.233 & -0.30 \\
\hline 0.3 & 0.0 & $4 / 3$ & 0.4 & & & & \\
\hline
\end{tabular}

\footnotetext{
${ }^{26}$ For this work, we assume that these two velocity dispersions are equal.
}

Table 1

The Scaling Parameters for Different Values of $\delta, \epsilon$, and $\alpha$

hence, we obtain the following relation of the power-law indices:

$$
\frac{\alpha}{\delta}=\frac{\epsilon}{\delta}-2+\frac{1}{\delta}
$$

This result shows that the scaling relations are fully determined by fixing the values for two of the parameters $\epsilon, \alpha$, and $\delta$. Thus, if we fix the $\epsilon$ range for the mass-to-light scaling we also fix the interval for the truncation scaling $\alpha$. The ratio of the elliptical galaxies' central dynamical mass and light is $M_{\mathrm{dyn}} / L \propto L^{\epsilon_{\mathrm{FP}}}$, with an FP slope of $\epsilon_{\mathrm{FP}} \approx 0.2$ (Bender et al. 1992). The exact value depends also on the filter used to measure the luminosity; see Barbera et al. (2011). Strong-lensing analyses that measure the central $M_{\text {tot }} / L$ also obtain a scaling of the central $M_{\text {tot }} / L \propto L^{\epsilon}$ with $\epsilon=0.2$ (see, e.g., Grillo et al. 2009; Auger et al. 2010). Weak-lensing analyses for field galaxies arrive at the same scaling for the total dark matter to light ratio (Brimioulle et al. 2013).

For halos in a dense environment, however, we expect the stripping radius to be (Merritt 1983)

$$
r_{\mathrm{t}} \propto M_{\mathrm{tot}}^{1 / 3}
$$

and with $M_{\text {tot }} \propto \sigma^{2} r_{\mathrm{t}}$, we obtain $\alpha / \delta=1$. The mass-velocity relation then becomes $M_{\text {tot }} \propto \sigma^{3}$. We use this relation, in tandem with Equation (6), to obtain the mass-to-light ratio:

$$
\frac{M_{\text {tot, stripped }}}{L} \propto L^{\epsilon_{\text {stripped }}} \propto \sigma^{3-\delta^{-1}} .
$$

And thus,

$$
\epsilon_{\text {stripped }}=3 \delta-1=3 \alpha-1 .
$$

Therefore, the power-law index of the mass-to-light ratio for stripped halos as a function of light is negative and the order of $\epsilon_{\text {stripped }}=-0.3$ to $\epsilon_{\text {stripped }}=-0.1$, depending on the value of $\delta$; see Table 1. In summary, we expect the value of $\epsilon$ to be between $\epsilon=0.2$ and $\epsilon=-0.3$, where the maximum and minimum values refer to the cases where no halo stripping has taken place and the case where halo stripping has been completed. MACSJ1206.2-0847 shows signs for both relaxation and thus completed halo stripping and for ongoing build up and thus still ongoing halo stripping. Therefore, we choose a value for the mass-to-light scaling between that for isolated field galaxies and the value expected for finalized stripping in the dense cluster center: we thus take $\epsilon=0$. Our choices for $\epsilon$ and $\delta$ lead to the following equation for the truncation scaling:

$$
\sigma=\sigma^{\star}\left(\frac{L}{L^{\star}}\right)^{0.3} \quad, \quad r_{\mathrm{t}}=r_{\mathrm{t}}^{\star}\left(\frac{\sigma}{\sigma^{\star}}\right)^{\frac{4}{3}} .
$$

This scaling relation between velocity dispersion and truncation radius is adopted in most parts of the paper. However, we also investigate whether the measurements of the halo sizes 
change if we assume $\delta=0.25$ and $\epsilon=0$. We find no significant changes in our results. Throughout this work, we assume Equation (14) (or its modification $\delta=0.25$ and $\epsilon=0$ ) to hold for all galaxies independent of the distance of the galaxy to the cluster center, i.e., a galaxy with velocity dispersion $\sigma^{\star}$ (and luminosity $L^{\star}$ ) always has a size of $r_{\mathrm{t}}^{\star}$. In this work, we only investigate the central, dense, strong-lensing region, meaning that we obtain an average truncation for all galaxies in the dense center. We cannot study truncation in less dense environments by extending the analysis done in this work to larger distances from the cluster center, since our analysis relies on the stronglensing effect. Instead, the analysis would have to be repeated in the centers of less dense clusters or groups of galaxies.

\section{STRONG LENSING MODEL FOR POINT-LIKE SOURCES}

The first redshift measurement of the giant arc, as well as the velocity dispersion and redshift of the brightest cluster galaxy (BCG), were reported by Sand et al. (2004). The first stronglensing model for cluster MACSJ1206.2-0847 was published by Ebeling et al. (2009), based on two SFB peaks multiply mapped into knots on the giant arc and its counterimage. The CLASH data allowed Zitrin et al. (2012b) to identify 12 multiply imaged systems lensed into 52 multiple images. Distances for the lensed galaxies were inferred from spectroscopic redshifts, if available, or precise photometric redshifts. In the following, we use a parametric strong-lensing model for the dark matter and the cluster members close to the strong-lensing area. We describe the model input first, followed by the results.

\subsection{Model Ingredients}

For the point-like strong-lensing analysis, we need two ingredients: the point-like multiple image positions and models for the cluster-scale mass distribution and its substructure as traced by the cluster galaxies.

\subsubsection{Multiple Image Systems}

We start with similar sources as Zitrin et al. (2012b, Table 1), but modify this selection. In Table 2, we present our multiple image identifications; their positions are shown in Figure 3. The differences from the Zitrin et al. (2012b) analysis are as follows: first, we keep systems 2, 3, 4, 5, 6, 7, 8, 12, and 13 unchanged. We split arc system 1 into 3 subsystems at the same redshift using the corresponding SFB peaks, labeled "1a," "1b," and "1c"; see also Figure 14. Since systems 2 and 3 are two brightness peaks in the same source, we replace these systems by numbers $2 b$ and 2c. For the systems 9 and 10, Zitrin et al. (2012b) state an ambiguity in the images 9.3,9.4, 10.3, and 10.4. We adopt these images as 10.3 and 10.4 only: first, the SFB distributions of 10.3 and 10.4 look more similar to 10.1 and 10.2 than to 9.1 and 9.2 and second, the best-fit model also gives a significantly better fit to this identification of the observations than 9.3 and 9.4. Also, for these systems, we neglect the only probable counterimages 9.5 and 10.5 of Zitrin et al. (2012b). For system 11, we also neglect the candidate images 11.1 and 11.2 , keeping 11.3 to 11.5 as a triple imaged system only. Our best-fit model does indeed not predict the multiple images 11.1 and 11.2 and gives model positions for 9.5 and 10.56 ".2 and 9'.5 away from the positions given in Zitrin et al. (2012b), respectively. However, there is no certain identification possible for these images.

We use the spectroscopic redshift of image systems measured as a part of a VIMOS campaign at the Very Large Telescope
Table 2

Multiple Image Positions

\begin{tabular}{|c|c|c|c|c|}
\hline $\begin{array}{l}\text { Obj. } \\
\text { ID }\end{array}$ & $\begin{array}{l}\Theta_{1}{ }^{a} \\
\left({ }^{\prime \prime}\right)\end{array}$ & $\begin{array}{l}\Theta_{2}{ }^{a} \\
\left({ }^{\prime \prime}\right)\end{array}$ & $z_{\text {input }}$ & $z_{\text {model }}$ \\
\hline 1a.1 & 12.85 & 19.73 & $1.033^{\mathrm{b}}$ & $1.033^{\mathrm{b}}$ \\
\hline 1a. 2 & 20.76 & 3.46 & $1.033^{\mathrm{b}}$ & $1.033^{\mathrm{b}}$ \\
\hline $1 \mathrm{a} .3$ & 19.56 & -6.79 & $1.033^{\mathrm{b}}$ & $1.033^{\mathrm{b}}$ \\
\hline 1b. 1 & 13.72 & 18.91 & $1.033^{\mathrm{b}}$ & $1.033^{\mathrm{b}}$ \\
\hline 1b. 2 & 20.71 & 4.96 & $1.033^{\mathrm{b}}$ & $1.033^{\mathrm{b}}$ \\
\hline 1b. 3 & 19.71 & -7.54 & $1.033^{\mathrm{b}}$ & $1.033^{\mathrm{b}}$ \\
\hline 1c. 1 & 12.46 & 20.26 & $1.033^{\mathrm{b}}$ & $1.033^{\mathrm{b}}$ \\
\hline $1 c .2$ & 19.56 & -5.84 & $1.033^{\mathrm{b}}$ & $1.033^{\mathrm{b}}$ \\
\hline $2 \mathrm{a} .1$ & -35.30 & -28.95 & $3.03^{\mathrm{b}}$ & $3.03^{\mathrm{b}}$ \\
\hline $2 \mathrm{a} .2$ & -42.15 & -14.20 & $3.03^{\mathrm{b}}$ & $3.03^{\mathrm{b}}$ \\
\hline $2 \mathrm{a} .3$ & -42.65 & 15.40 & $3.03^{\mathrm{b}}$ & $3.03^{\mathrm{b}}$ \\
\hline 2b. 1 & -33.60 & -30.95 & $3.03^{\mathrm{b}}$ & $3.03^{\mathrm{b}}$ \\
\hline $2 b .2$ & -42.15 & -12.85 & $3.03^{\mathrm{b}}$ & $3.03^{\mathrm{b}}$ \\
\hline $2 b .3$ & -42.30 & 14.65 & $3.03^{\mathrm{b}}$ & $3.03^{\mathrm{b}}$ \\
\hline $2 c .1$ & -34.00 & -30.45 & $3.03^{\mathrm{b}}$ & $3.03^{\mathrm{b}}$ \\
\hline $2 c .2$ & -42.11 & -13.15 & $3.03^{\mathrm{b}}$ & $3.03^{\mathrm{b}}$ \\
\hline $2 c .3$ & -42.30 & 14.85 & $3.03^{\mathrm{b}}$ & $3.03^{\mathrm{b}}$ \\
\hline 4.1 & 14.37 & 12.57 & $2.54^{\mathrm{b}}$ & $2.54^{\mathrm{b}}$ \\
\hline 4.2 & -6.43 & 21.42 & $2.54^{\mathrm{b}}$ & $2.54^{\mathrm{b}}$ \\
\hline 4.3 & -15.10 & 2.74 & $2.54^{\mathrm{b}}$ & $2.54^{\mathrm{b}}$ \\
\hline 4.4 & 0.62 & 3.63 & $2.54^{\mathrm{b}}$ & $2.54^{\mathrm{b}}$ \\
\hline 4.5 & 6.36 & -39.21 & $2.54^{\mathrm{b}}$ & $2.54^{\mathrm{b}}$ \\
\hline 5.1 & -21.60 & 17.60 & $1.73 \pm 0.17^{\mathrm{c}}$ & 1.59 \\
\hline 5.2 & -22.30 & -2.80 & $1.73 \pm 0.17^{\mathrm{c}}$ & 1.59 \\
\hline 5.3 & -6.50 & -30.45 & $1.73 \pm 0.17^{\mathrm{c}}$ & 1.59 \\
\hline 6.1 & 13.95 & 28.15 & $2.73 \pm 0.15^{\mathrm{c}}$ & 1.86 \\
\hline 6.2 & 22.36 & -23.50 & $2.73 \pm 0.15^{\mathrm{c}}$ & 1.86 \\
\hline 6.3 & 26.25 & 11.30 & $2.73 \pm 0.15^{\mathrm{c}}$ & 1.86 \\
\hline 7.1 & -56.30 & -15.10 & $3.82 \pm 0.3^{c}$ & 2.90 \\
\hline 7.2 & -55.60 & -19.30 & $3.82 \pm 0.3^{\mathrm{c}}$ & 2.90 \\
\hline 7.3 & -53.10 & -24.30 & $3.82 \pm 0.3^{\mathrm{c}}$ & 2.90 \\
\hline 7.4 & -56.29 & -13.62 & $3.82 \pm 0.3^{\mathrm{c}}$ & 2.90 \\
\hline 7.5 & -56.61 & -12.68 & $3.82 \pm 0.3^{c}$ & 2.90 \\
\hline 8.1 & -2.67 & 34.72 & $5.46 \pm 0.29^{c}$ & 5.03 \\
\hline 8.2 & 23.27 & 13.86 & $5.46 \pm 0.29^{c}$ & 5.03 \\
\hline 8.3 & -16.33 & -0.46 & $5.46 \pm 0.29^{c}$ & 5.03 \\
\hline 8.4 & 13.01 & -40.68 & $5.46 \pm 0.29^{c}$ & 5.03 \\
\hline 9.1 & 8.95 & 14.05 & $1.73 \pm 0.23^{\mathrm{c}}$ & 1.64 \\
\hline 9.2 & 2.40 & 16.55 & $1.73 \pm 0.23^{\mathrm{c}}$ & 1.64 \\
\hline 10.1 & 0.35 & 18.95 & $1.34 \pm 0.26^{\mathrm{c}}$ & 1.69 \\
\hline 10.2 & 12.30 & 10.70 & $1.34 \pm 0.26^{\mathrm{c}}$ & 1.69 \\
\hline 10.3 & -5.55 & 2.00 & $1.34 \pm 0.26^{\mathrm{c}}$ & 1.69 \\
\hline 10.4 & -2.45 & 2.25 & $1.34 \pm 0.26^{\mathrm{c}}$ & 1.69 \\
\hline 11.3 & -10.79 & 19.02 & $1.35 \pm 0.44^{\mathrm{c}}$ & 1.44 \\
\hline 11.4 & -13.87 & -0.56 & $1.35 \pm 0.44^{\mathrm{c}}$ & 1.44 \\
\hline 11.5 & 2.38 & -28.57 & $1.35 \pm 0.44^{\mathrm{c}}$ & 1.44 \\
\hline 12.1 & -19.04 & 33.42 & $3.84 \pm 0.52^{\mathrm{c}}$ & 3.28 \\
\hline 12.2 & -24.78 & -7.58 & $3.84 \pm 0.52^{c}$ & 3.28 \\
\hline 12.3 & -3.95 & -36.07 & $3.84 \pm 0.52^{\mathrm{c}}$ & 3.28 \\
\hline 13.1 & -10.99 & -37.61 & $3.18 \pm 0.99^{c}$ & 2.34 \\
\hline 13.2 & -29.83 & -1.72 & $3.18 \pm 0.99^{c}$ & 2.34 \\
\hline 13.3 & -28.73 & 17.18 & $3.18 \pm 0.99^{c}$ & 2.34 \\
\hline
\end{tabular}

Notes.

${ }^{\text {a }}$ Relative to the center of the BCG at 12:06:12.134 R.A. (J2000), -08:48:03.35 decl. (J2000).

b Spectroscopic redshift, fixed.

${ }^{c}$ Photometric redshift estimate, weighted mean and error. 


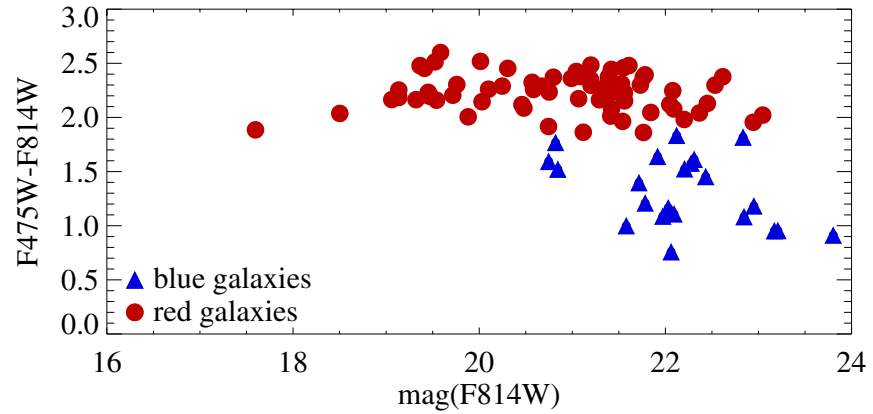

Figure 1. Color-magnitude diagram of the selected cluster galaxy lenses. F475W-F814W color is plotted against the F814W magnitude of the galaxies. We mostly select red galaxies with similar color. Since we do not select by galaxy color but instead by photometric and spectroscopic redshift, we also identify some bluer galaxies as cluster members, which would not have been possible based on a pure red sequence cut. The typical error on the magnitude and color is smaller than the symbol size. The symbol indicates the SED type of the galaxies, separated into red and blue galaxies.

(A color version of this figure is available in the online journal.)

(VLT), when these are available. Otherwise, we combine the available photometric redshifts in Zitrin et al. (2012b) into an error weighted mean redshift and mean error for each multiple image system belonging to the same source. The mean redshift becomes the central value for a Gaussian-shaped redshift prior, and the mean redshift error becomes the $1 \sigma$ width of this prior. This technique provides an approximate, more conservative estimate for the uncertainties of the redshifts than the rms error of the mean. Any systematic uncertainty in the photometric redshift estimate is equally present in the estimate of each multiple image, since they have the same color. Therefore, a pure statistical error would underestimate the true uncertainty of the photometric redshift. These photometric redshift constraints of the multiple image systems are used as priors in the model optimization.

We adopt a value of 0.5 for the positional uncertainty of the multiple images. This value is driven by line-of-sight (LOS) structure and substructure not accounted for in the lens modeling, since the measurement error of the positions of the multiple images is usually only a fraction of a pixel. Jullo et al. (2010) estimate the LOS structure to produce an rms image position scatter of $\approx 1^{\prime \prime}$ for a cluster like A1689. Host (2012) estimates a relative LOS structure deflection angle depending on the distance from the cluster center and the redshift of the source to be $0.5-2$ '.5 for typical strong-lensing situations.

\subsubsection{Cluster Galaxies Tracing Dark Matter Substructure}

We use the Bayesian photometric redshifts (Benítez 2000; Benítez et al. 2004; Coe et al. 2006) as described in Postman et al. (2012) and spectroscopic information for this cluster (P. Rosati et al. 2013, in preparation) wherever available for the cluster member selection. For simplicity, we consider as cluster members galaxies with spectroscopic redshifts between $z=0.43$ and 0.45; all other galaxies with spectroscopic redshifts, outside of this range are excluded.

For galaxies lacking spectroscopic redshifts, we use photometric redshift estimates and consider all galaxies with a bestfitting photometric redshift estimate between 0.39 and 0.49 and a $95 \%$ confidence interval width smaller than 0.5 (i.e., c.1. $(95 \%)_{\max }-$ c.1. $\left.(95 \%)_{\min }<0.5\right)$ as cluster members as well. From these cluster galaxies, we use only a subsample that fulfill two criteria: first, we only use those within a $3^{\prime} \times 3^{\prime}$ sized box centered on the BCG to cover the strong-lensing area only.

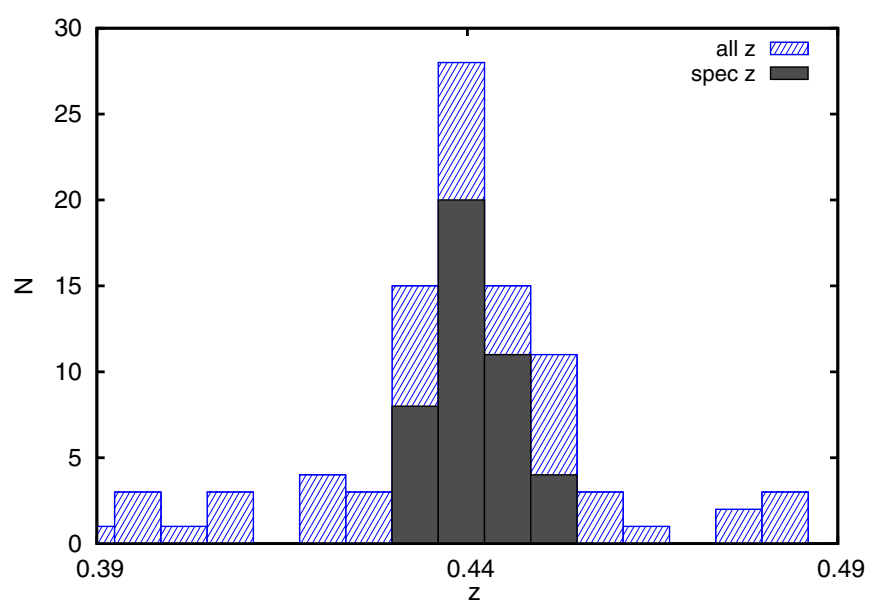

Figure 2. Redshift distribution of the modeled cluster members. The spectroscopically selected members are drawn as the solid histogram; all selected members are shown as the hatched distribution. As can be seen, both distributions peak at $z \sim 0.44$, giving the redshift of the cluster.

(A color version of this figure is available in the online journal.)

Second, these galaxies have to trace a sufficiently massive halo to be relevant for the lens modeling: from the galaxy sample, we pick the second brightest galaxy of this cluster, located at 12:06:15.647 R.A. (J2000), -08:48:21.88 decl. (J2000) as the reference galaxy (called hereafter GR); see Figure 3. We use the F160W fluxes of the cluster members in units of the GR and use Equation (6) to scale the velocity dispersions relative to the GR.

We convert the velocity dispersions in a "cosmology-free" Einstein radius by

$$
\Theta_{\mathrm{E}}=\frac{4 \pi \sigma^{2}}{c^{2}},
$$

where $c$ is the vacuum speed of light. We explicitly model only those cluster galaxies that have an Einstein radius larger than $3 \%$ of the Einstein radius of the GR, meaning that we neglect galaxies with an Einstein radius smaller than $\sim 1$ pixel. The redshift distribution of the cluster members selected finally, split into galaxies selected spectroscopically and photometrically, is plotted in Figures 1 and 2. In both subgroups, the cluster is clearly visible as one peak at a redshift $z=0.44$. The cluster members form a red sequence in color-magnitude space, as seen in Figure 1, with a minor fraction of galaxies being classified as blue. The distribution of these galaxies in color-magnitude space is shown in Figure 1.

For the selected cluster members, an Einstein radius of $1^{\prime \prime}$ corresponds to a velocity dispersion $\sigma=186 \mathrm{~km} \mathrm{~s}^{-1}$. Based on Equation (14), we note that we need to measure two values to fully determine the halo properties: $\sigma^{\star}$ and $r_{\mathrm{t}}^{\star}$. We use two different sets of parameters: $r_{\mathrm{t}, 1^{\prime \prime}}$, for a reference $\sigma=186 \mathrm{~km} \mathrm{~s}^{-1}$, which gives the value for a galaxy with an Einstein radius of $\Theta_{\mathrm{E}}=1^{\prime \prime}$, and $r_{\mathrm{t}, \mathrm{GR}}$, which gives the truncation radius for the GR itself.

With this procedure, we obtain 92 galaxies. We measure their positions, orientations, and ellipticities from SEXTRACTOR (Bertin \& Arnouts 1996) run on the HST/ACS F606W band. A list of all cluster galaxies in our model is provided in Table 9. A comparison with the HST/ACS F814W data shows consistent values for the orientations and ellipticities of the cluster members.

With Equations (6) and (14), we now have a complete description of all cluster galaxy lenses with only two free parameters (the normalizations of Equations (6) and (14)). Since 
Table 3

The Model Lens Input Parameters and Priors

\begin{tabular}{lcccc}
\hline \hline Parameter & Prior & Min & Max & Model Result (95\% c.1.) \\
\hline$\gamma$ & Uniform & 0 & 0.4 & $0.20_{-0.03}^{+0.03}$ \\
$\Theta_{\gamma}$ & Uniform & $-90^{\circ}$ & $90^{\circ}$ & $25.7_{-2.5}^{+3.0 \circ}$ \\
$x_{\mathrm{NFW}}{ }^{\mathrm{a}}$ & Uniform & $-8^{\prime \prime}$ & $8^{\prime \prime}$ & $0.19_{-0.47}^{+0.44}$ \\
$y_{\mathrm{NFW}}{ }^{\mathrm{a}}$ & Uniform & $-8^{\prime \prime}$ & $8^{\prime \prime}$ & $0.78_{-0.23}^{+0.23}$ \\
$q_{\mathrm{NFW}}$ & Uniform & 0.35 & 1 & $0.686_{-0.016}^{+0.014}$ \\
$\Theta_{\mathrm{NFW}}$ & Uniform & $-20^{\circ}$ & $44^{\circ}$ & $19.0_{-1.0}^{+1.2 \circ}$ \\
$\Theta_{\mathrm{E}, \mathrm{NFW}}$ & Uniform & $25^{\prime \prime}$ & $200^{\prime \prime}$ & $43.8_{-1.4}^{+1.2}$ \\
$r_{\mathrm{s}, \mathrm{NFW}}$ & Uniform & $50^{\prime \prime}$ & $650^{\prime \prime}$ & $175_{-20}^{+23}$ \\
$r_{\mathrm{t}, 1^{\prime \prime}}$ & Uniform & $11 \mathrm{kpc}^{\prime \prime}$ & $142 \mathrm{kpc}^{\prime \prime}$ & $31_{-14}^{+36} \mathrm{kpc}$ \\
$\sigma_{\mathrm{GR}}$ & Uniform & $59 \mathrm{~km} \mathrm{~s}^{-1}$ & $395 \mathrm{~km} \mathrm{~s}^{-1}$ & $236_{-32}^{+29} \mathrm{~km} \mathrm{~s}^{-1}$ \\
\hline
\end{tabular}

Notes. The model lens input parameters and priors are stated. Given are the parameter, its prior type, the minimal and maximal allowed value, as well as the most likely value and its $95 \%$ c.l. error.

${ }^{\text {a }}$ Relative to the center of the BCG at 12:06:12.134 R.A. (J2000), -08:48:03.35 decl. (J2000).

we take $L^{\star}$ for the galaxy GR, the only free parameters in our galaxy model are $\sigma_{\mathrm{GR}}$, thus fully determining Equation (6), and $r_{\mathrm{t}, \mathrm{GR}}$, fully determining Equation (14) for $\sigma_{\mathrm{GR}} \cdot{ }^{27} \mathrm{We}$ will attribute these two parameters to the GR, but we should, however, keep in mind that the derived parameters of the GR are due to the combined signal of all the galaxies and that it is irrelevant which galaxy was chosen as the reference. For the GR, we consistently measure an effective radius $R_{\text {eff }}$ of $5 \mathrm{kpc}-6 \mathrm{kpc}$ from fitting a Sérsic, (Sérsic 1963), a de Vaucouleurs (de Vaucouleurs 1948), and a de Vaucouleurs + exponential disk model in the F160W and F814W filters using GALFIT (Peng et al. 2010). This effective radius agrees well with measurements (in the HST-F814W and VLT-FORS-I-band filters) of other elliptical galaxies in various clusters of similar redshift; see Figure 10 in Saglia et al. (2010).

\subsubsection{Modeling of the Cluster Component}

We model the cluster as an NFW (Navarro et al. 1997) halo. We also tried a non-singular isothermal elliptical (NSIE) profile for the halo, but doing so results in worse fits to the positions of the multiple image systems. The best-fit $\chi^{2}$ for the NFW profile is $\chi_{\mathrm{NFW}}^{2}=227$, while an NSIE cluster-scale halo with the same number of free parameters gives a $\chi_{\mathrm{NSIE}}^{2}=434$, for the full model using point-like images. A similar difference for an NSIE versus NFW model has been reported already for the stacked weak-lensing signal of clusters and groups of galaxies in the SDSS (Mandelbaum et al. 2006).

We also add external shear as a free parameter to allow for a contribution of the large-scale environment in the vicinity of the cluster.

This gives in total six free parameters for the NFW halo, 2 for the external shear, 2 for the galaxy lenses, 9 for the source redshifts, and 32 for the (R.A., decl.) source positions of the 16 sources. The lens model parameters and its priors are listed in Table 3. We use uniform priors with defined minimum and maximum values for each of the parameters. From the multiple images, we obtain 104 constraints, leaving this model with 53 degrees of freedom.

\footnotetext{
27 However, we can equivalently use $r_{\mathrm{t}, 1^{\prime \prime}}$ with $\sigma=186 \mathrm{~km} \mathrm{~s}^{-1}$ as the full determination of Equation (14).
}

\subsection{Results of the Point-like Modeling}

Putting everything together, we can now reconstruct the lensing signal for this cluster. We use the strong-lensing code GLEE, a lens modeling software developed by S. H. Suyu and A. Halkola (Suyu \& Halkola 2010; Suyu et al. 2012). This method not only yields the best-fitting model (using either source plane or image plane minimization) but also includes a Monte Carlo Markov Chain (MCMC) sampler yielding the most likely parameters with their confidence limits. We obtain the best-fitting cluster model by maximizing the posterior probability distribution function. For that, the likelihood is multiplied by the priors; see Halkola et al. (2006, 2008) and Suyu \& Halkola (2010). The likelihood is proportional to $\sim \exp \left(-\chi^{2} / 2\right)$. The $\chi^{2}$ is calculated from the difference between the observed and the modelpredicted image position:

$$
\chi^{2}=\sum_{i} \frac{\left\|\boldsymbol{\Theta}_{i}-\boldsymbol{\Theta}_{0, i}\right\|^{2}}{\delta_{\Theta_{i}}^{2}},
$$

where $\boldsymbol{\Theta}_{i}$ and $\boldsymbol{\Theta}_{0, i}$ mark the model-predicted and observed position of the $i$ th multiple image, respectively; $\delta_{\Theta_{i}}$ is its input uncertainty. The MCMC sampling procedure is described in Dunkley et al. (2005) and Suyu \& Halkola (2010). We obtain acceptance rates of typically $\sim 0.25$ for the MCMC; the covariance matrix between parameters is derived from a previous run of the MCMC procedure for the same model parameters. Convergence is achieved based on the power spectrum test given in Dunkley et al. (2005).

\subsubsection{Results for the Cluster-scale Model}

For the best-fit values, ${ }^{28}$ we obtain: $r_{\mathrm{t}, 1^{\prime \prime}}=23.7 \mathrm{kpc}$, $\sigma_{\mathrm{GR}}=246 \mathrm{~km} \mathrm{~s}^{-1}, \gamma=0.19, \Theta_{\gamma}=26^{\circ}, x_{\mathrm{NFW}}=0^{\prime \prime} 15$, $y_{\mathrm{NFW}}=0.74, b / a_{\mathrm{NFW}}=0.69, \Theta_{\mathrm{NFW}}=19^{\circ}, \Theta_{\mathrm{E}, \mathrm{NFW}}=44^{\prime \prime} .1$, and $r_{\mathrm{s}, \mathrm{NFW}}=174^{\prime \prime}$. As explained already, the external shear and the Einstein radius are given in units of $D_{\mathrm{ds}} D_{\mathrm{s}}^{-1}$. The redshift estimates of the best-fit model are given in Table 2. Most of the redshifts agree with their photometric estimates within the errors; only system 6 is a clear outlier. The critical lines for the arc redshift and a redshift of $z=2.54$ are plotted in Figure 3.

In Figure 4, we show the differences between the input and model output positions for our best-fit model. As one can see, the mean and median differences are 0.86 and $00^{\prime \prime} 82$, respectively. These results justify the use of the input uncertainty of 0 '. 5 , since this is a good estimate of the reconstruction uncertainty. The MCMC sampling provides us with estimates of the parameter uncertainties.

The probability densities for the parameter estimates are shown in Figure 5. We wish to discuss some of the parameters here, quoting the $95 \%$ confidence intervals: first, the external shear values are $\gamma=0.20_{-0.03}^{+0.03}$ and $\Theta_{\gamma}=25.7_{-2.5}^{+3.0 \circ}$. This shear can originate from external structure present in the vicinity of the cluster or from substructure present in the cluster, but not accounted for in the model. Indeed, the cluster mass reconstruction map of Umetsu et al. (2012; see their Figure 8) shows two additional structures, one in the southeast and the other in the northwest of the cluster center. We take the 2D mass reconstruction map of Umetsu et al. (2012) and subtract the surface mass density of their best-fitting cluster NFW profile, leaving us with the residual mass map. We calculate the shear that these additional masses cause in the cluster center, and

\footnotetext{
28 The error estimates from the MCMC sample will be discussed below.
} 


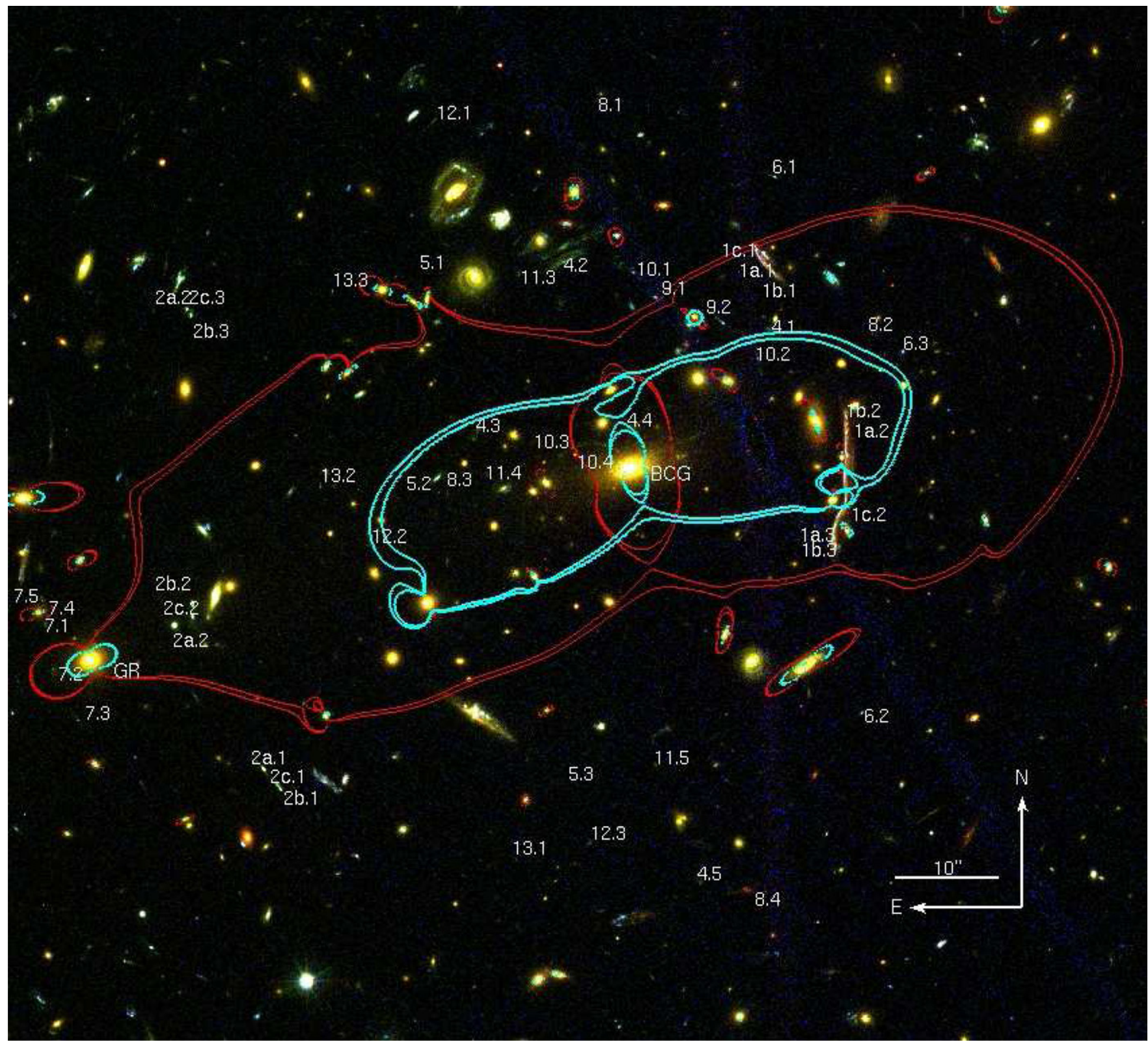

Figure 3. $110^{\prime \prime} \times 100^{\prime \prime}$ cutout of the cluster center. The multiple image systems are labeled according to Table 2 . We have added the critical lines for a source at the redshift of the arc $(z=1.03)$ in cyan and for a source at $z=2.54$ in red. The critical lines are calculated from a pixelated magnification map, enclosing the high magnification areas of the image. The BCG and the GR are marked in the image. North is up and east is to the left. This color composite image is made from F435W, F606W, and F814W HST/ACS filter data.

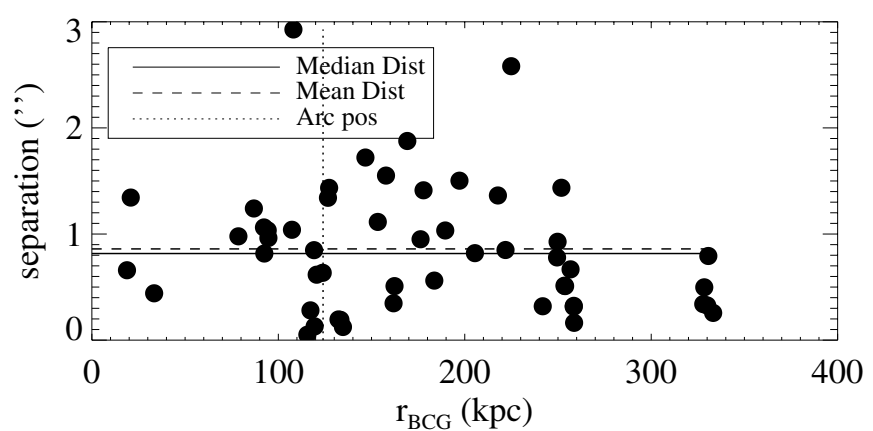

Figure 4. Distance between observed and model predicted multiple image position vs. distance from the center of the BCG. Overplotted are the respective median and mean of the images. The vertical dotted line marks the mean distance of the giant arc and its counterimage to the center of the BCG. There is no radial dependence of the error visible in this model. obtain values of $\gamma \lesssim 0.13$ for $D_{\mathrm{ds}} D_{\mathrm{s}}^{-1}=1$. This external structure thus explains a part of the shear present in the model. Additional or external shear can in principle be produced by any mass distribution that we do not model explicitly. The mass distribution associated with the intracluster light is such a component: it ranges from the BCG toward the GR (in the southeast) and beyond the GR (see Figure 6). We tested that the presence of this intracluster light is not a superposition of the light associated with the cluster members: we subtracted a galaxy light model for the galaxies in the southeast from the F160W data; the residual light is not centered on any galaxy halos and hence it cannot be attributed to a galaxy. The gravitational shear produced by the mass associated with the intracluster light is incorrectly attributed to the external shear if we do not explicitly model its lensing contribution, and thus increases the external shear of the lensing model. We 


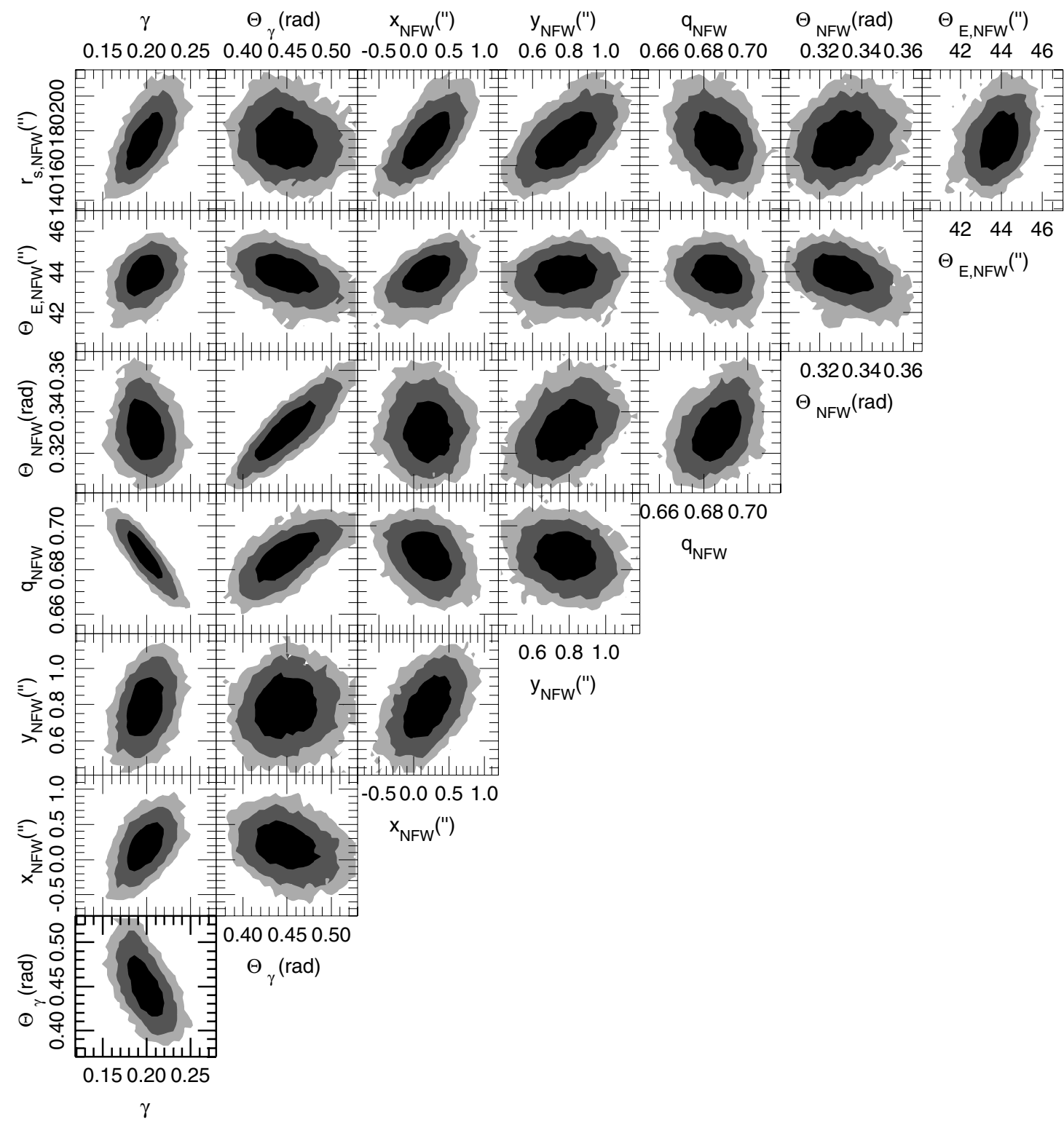

Figure 5. Parameter estimates from the MCMC sampling of the parameter space. The shaded regions give the $68.3 \%, 95.4 \%$, and $99.7 \%$ uncertainty areas, from dark to light gray, respectively.

employ a test scenario, explicitly modeling a mass distribution associated with the intracluster light. We used a non-singular, highly elongated $(q<0.4)$ isothermal ellipsoid with a large core radius and a small truncation radius that roughly resembles a mass bar. The best-fit masses of this intra-stellar light component are modest (a few times $10^{12} M_{\odot}$ ). The external shear values required in this toy model drop to $\gamma=0.13_{-0.04}^{+0.04}$, agreeing with our estimate based on Umetsu et al. (2012). We verify that this toy model (approximately including the intracluster light) results in the same sizes of galaxies as the strong-lensing model presented in this work.

Second, the cluster-scale NFW halo has the following most likely parameter estimates: $x_{\mathrm{NFW}}=0.19_{-0.47}^{+0.44}{ }^{\prime \prime}, y_{\mathrm{NFW}}=$ $0.78_{-0.23}^{+0.23} ", q_{\mathrm{NFW}}=b / a_{\mathrm{NFW}}=0.686_{-0.016}^{+0.014}, \Theta_{\mathrm{NFW}}=19.0_{-1.0}^{+1.2 \circ}$, $\Theta_{\mathrm{E}, \mathrm{NFW}}=43.8_{-1.4}^{+1.2}$, and $r_{\mathrm{s}, \mathrm{NFW}}=175_{-20}^{+23}{ }^{\prime \prime}$. The results regarding the cluster-scale dark matter halo are within our expectations.

1. The halo center's position follows the same trend as the X-ray center in Ebeling et al. (2009), i.e., the center has a slight tendency to move toward positive values of $x$ and $y$ relative to the BCG center. In total, the center of mass is shifted by approximately $(0.8 \pm 0.3)^{\prime \prime}$. Ebeling et al. (2009) report a displacement of the X-ray center from the BCG center of $1^{\prime \prime} .7 \pm 0.4$ in approximately the same direction, implying that these displacements agree at a $2 \sigma$ level. The level of displacement between the BCG and the dark matter halo center is comparable to the results of Zitrin et al. (2012a).

2. The orientation of the NFW major axis follows the major axis of the $\mathrm{BCG}$ to within $\approx 5^{\circ}$.

3. There is some degeneracy between the orientation of the cluster halo and the external shear, since both can compensate each other partially. The same is true for the axis ratio of the halo and the value of the external shear.

4. For the Einstein and scale radius of the NFW halo, we obtain $\Theta_{\mathrm{E}, \mathrm{NFW}}=43.8_{-1.4}^{+1.2}$ " and $r_{\mathrm{s}, \mathrm{NFW}}=175_{-20}^{+23}$ ". The total mass included within a cylinder of radius $R$ is shown in Figure 7. Our results for $M(<R)$ agree well with previous results obtained with various methods and presented in Umetsu et al. 


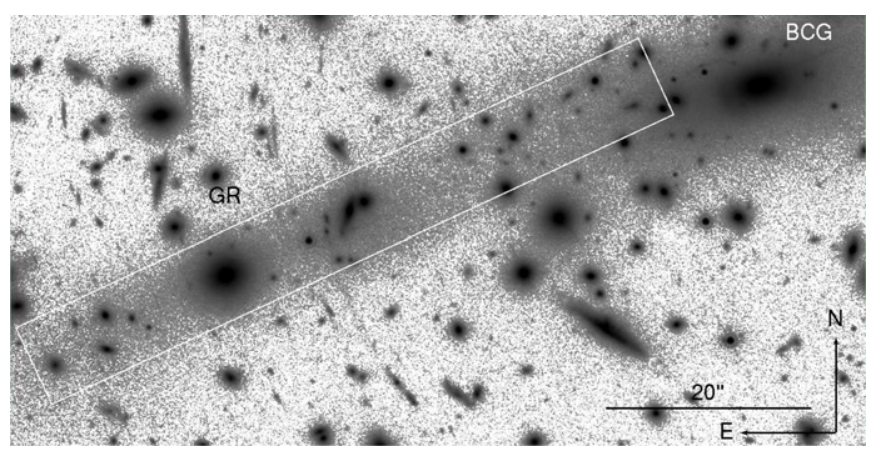

Figure 6. Center of the cluster MACSJ1206.2-0847 as observed with the F160W HST/WFC3 filter. The faint, bar-like structure in the intracluster light is marked with a white box. It extends $\sim 1$ '.5 radially outward from the BCG to the southeast. The mass associated with this intracluster light acts as further substructure. We used logarithmic scaling for the fluxes in this image.

(2012); see their Figure 7. The agreement holds up out to $\approx 300 \mathrm{kpc}$, which equals the radius probed by multiple images in this cluster. The result from "Zitrin MCMC" 29 agrees within its errors with all further results shown in Umetsu et al. (2012). Since this result is furthermore the only strong-lensing result in this work with realistic errors, we only compare to "Zitrin MCMC" below. Our errors on the measured masses are derived from the mass distribution of 200 random cluster models from the MCMC points. Since we use a parametric model for the lens, we only measure the uncertainty within this parametric model, and do not take into account the fact that different parameterizations could give similarly good fits with a slightly different mass profile. Hence, we are underestimating the true error on the radial mass profile. To obtain more realistic errors, we could take the same approach as in Umetsu et al. (2012) for the "Zitrin MCMC" results, thereby increasing our errors by the amount of the difference between the Zitrin et al. (2012b) and the "Zitrin MCMC" results. Our result (black area in Figure 7), however, already now agrees within the errors with that of the "Zitrin MCMC" findings (blue area, Figure 7). Since the results of the strong-lensing analysis of Zitrin et al. (2012b) and its improvement in Umetsu et al. (2012) have been presented in detail, we summarize here the differences with our method. In Zitrin et al. (2012b), both the mass associated with cluster members and the dark matter of the cluster are modeled starting from the light distribution of the cluster. The mass associated with the cluster members is obtained by scaling the galaxy masses with their light and modeling their mass density profile with a power law (two parameters). The dark matter of the cluster is obtained from smoothing the galaxy light (one further parameter) and scaling this value to the dark matter leaving amplitude as one further free parameter. In addition, there are two free parameters for external shear. By construction, this method does not allow for any dark matter not traced by galaxy light. Also, the radial dark matter profile is closely linked to the cluster light profile, since any deviation from that can only be achieved by smoothing. If the concentration of the cluster light profile obtained from the smoothed galaxy light is different from the concentration of the dark matter, a systematic error on the mass estimate and a bias in the determination of the true dark matter concentration can result. At least for the

29 See below for the explanation of this wording.

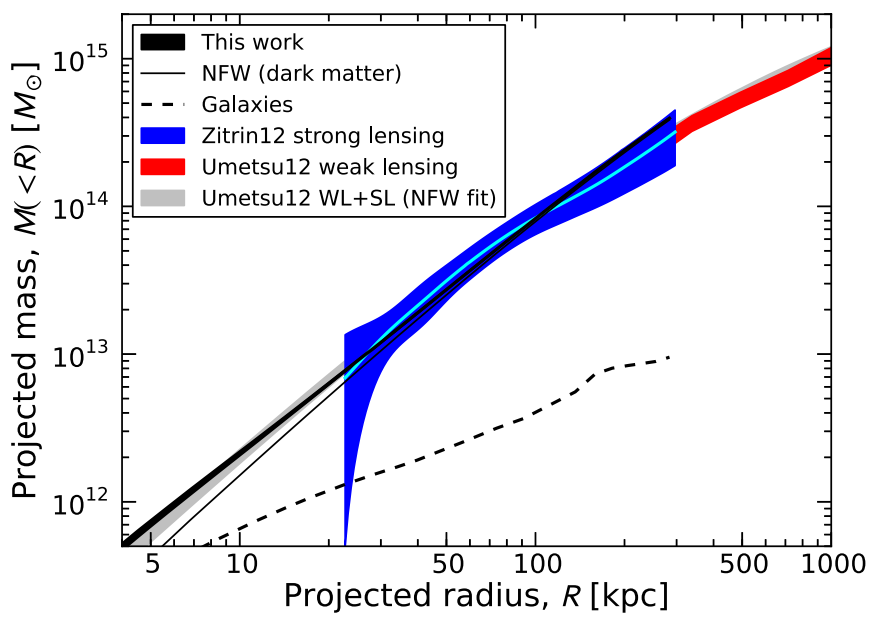

Figure 7. Projected mass estimates within circular apertures. The black area shows the $68 \%$ confidence interval for the combined mass, and the black solid and dashed lines show the mass contributions for the NFW halo alone and the galaxies for the best-fit model, respectively. The small uncertainty on the mass estimate comes from the fact that we use a parametric model, which needs to reproduce the correct Einstein radius, therefore giving too small errors for intermediate radii. We overplot the mass estimates from Umetsu et al. (2012), more explicitly their NFW fit to the weak- and strong-lensing data, their weaklensing mass estimates alone, and the Zitrin et al. (2012b) and Umetsu et al. (2012) strong-lensing estimate. In cyan (dark gray), the best-fitting estimate from Umetsu et al. (2012) for the same strong-lensing model is shown in cyan. The mass estimate in this work agrees in the range of $\sim 4 \mathrm{kpc}$ to $\sim 150 \mathrm{kpc}$ with our previous work.

(A color version of this figure is available in the online journal.)

number density distribution of cluster members, this seems to be indeed the case: Budzynski et al. (2012) find that the number density profile of cluster members of SDSS clusters follows an NFW profile but with a factor of two lower concentration than in the dark matter (independent of the mass of the cluster). In Umetsu et al. (2012), the method of Zitrin et al. (2012b) has been generalized by allowing the mass associated with the BCG to be modeled separately. In addition, Umetsu et al. (2012) altered the covariance matrix such that error estimates are increased to account for the too small systematic errors inherent in a parametric reconstruction. This improved analysis relative to the Zitrin et al. (2012b) analysis is called "Zitrin MCMC" in Umetsu et al. (2012).

Our method is different: we use a parameterized model for a cluster-scale lens, including it explicitly as an elliptical NFW profile (two main free parameters for the concentration and the virial radius, two free parameters for the ellipticity and major axis angle, and, in principle, two free parameters to locate the center of mass (the center of mass from the modeling in this cluster, however, is similar to the BCG). The galaxy-scale mass component is parameterized by two free parameters (halo depth and halo size). So, formally, our method has slightly more free parameters than that of Zitrin et al. (2012b) and Umetsu et al. (2012). Both methods are complementary as our method allows the placement of halos even if there is no light tracing them (or allows halos to be off center from their light), whereas the Zitrin et al. (2012b) method allows for small-scale variations in the dark matter, which however are linked to a smoothed version of the light. As far as the galaxy matter component is concerned, our method describes galaxies as being isothermal out to large radii (as obtained from strong-lensing and weak-lensing analyses of red galaxies; 
see Gavazzi et al. 2007; Auger et al. 2010) and allows for a cutoff (smaller than for field galaxies). In contrast, Zitrin et al. (2012b) can, once having tied the central matter density of galaxies to their central light, only change the total mass associated with galaxies by changing their matter density power-law slope. This picture seems to be an inaccurate description when tidal stripping of halos is described, since tidal stripping is not expected to change the central properties, but rather to shrink the halos from the outside in (Gao et al. 2004a). The precision that can be obtained with our method is superior; the image plane reproduction error is $1^{\prime \prime} .76$ in Umetsu et al. (2012) whereas it is 0'.85 in our work. This is likely the case not because of the increased number of free parameters, but rather because the galaxy component is modeled in a better way. Our approach for modeling the galaxy component is also followed by Zitrin et al. (2013) in their strong-lensing model for the mass distribution of MACSJ0416.1-2403. Zitrin et al. (2013) also compare the performance for a cluster component obtained with a mass follows light approach with an elliptical NFW component (leaving the galaxy component the same), finding that the latter provides the better fit.

5. We fit a circular $\mathrm{NFW}^{30}$ halo to the total azimuthally averaged mass in Figure 7 to estimate the concentration $c_{200}$ and $r_{\mathrm{s}, \mathrm{NFW}}$ from the total included mass with a least-squares fit. We obtain a concentration of $c_{200}=3.7 \pm 0.2$ and a scale radius of $r_{\mathrm{s}, \mathrm{NFW}}=677 \pm 48 \mathrm{kpc}$. When we exclude the central $70 \mathrm{kpc}$ from the fit, we obtain $c_{200} \approx 3.2$ and $r_{\mathrm{s}, \mathrm{NFW}}=827 \mathrm{kpc}$. Our radially averaged mass distribution agrees with the results of Umetsu et al. (2012) in the center. Our scale radius value is an extrapolation beyond the scales of strong-lensing data points. Since Umetsu et al. (2012) do a combined strong- and weak-lensing analysis constraining the profile on a much larger scale than our work can, their confidence intervals for these two parameters are smaller than ours and their conclusions are much more robust. Regarding the results of MACSJ1206.20847's mass-concentration relation, we therefore refer the reader to the work of Umetsu et al. (2012).

\subsubsection{Results for Galaxy Halos Tracing the Cluster Substructure}

Using the F160W flux of the galaxies and the scaling relations, the mass distribution of the galaxies is described as a function of two free parameters, the velocity dispersion of the GR, $\sigma_{\mathrm{GR}}$, and the normalization of the truncation radius scaling, $r_{\mathrm{t}, 1^{\prime \prime}}$. This truncation scale $r_{\mathrm{t}, 1^{\prime \prime}}$ is not to be confused with $r_{\mathrm{t}, \mathrm{GR}}$, which gives the truncation radius for the GR and is shown in Figure 9. For these two values, we obtain most likely values of $r_{\mathrm{t}, \mathrm{GR}}=41_{-18}^{+34} \mathrm{kpc}$ and $\sigma_{\mathrm{GR}}=236_{-32}^{+29} \mathrm{~km} \mathrm{~s}^{-1}$.

We apply the FJ relation and show the velocity dispersions for all cluster members galaxies as a histogram in Figure 8. Since the lenses' impacts scale as $\propto \sigma^{2}$, most of the low velocity dispersion galaxies have a minor influence on the lensing signal. There is, however, a secondary effect, i.e., that the deflection angle that a galaxy can impose on the LOS to a multiple image position depends also on the transverse distance to it. We therefore now weight each cluster galaxy by the mean deflection angle it imposes on all multiple images and obtain the effective velocity dispersion histogram for the cluster members, also shown in Figure 8. This figure shows that

\footnotetext{
30 We give the values for an overdensity of $\Delta=200$. The conversion to Umetsu et al. (2012), who use $\Delta=132$, is $c_{132} \sim 1.2 c_{200}$.
}

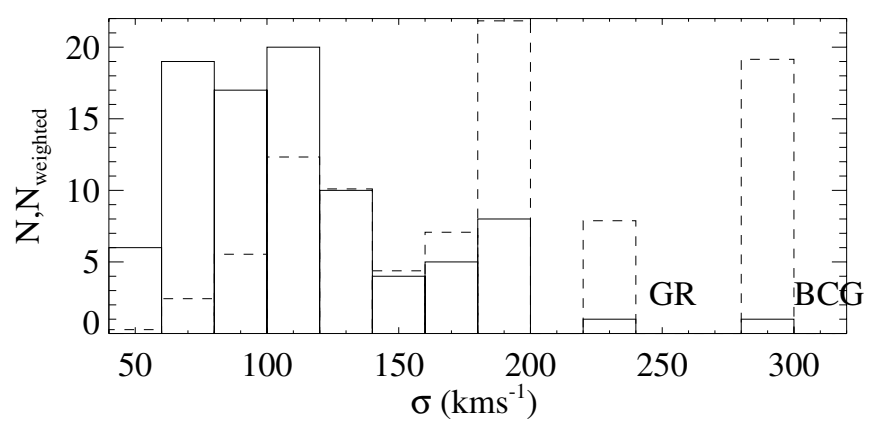

Figure 8. Velocity dispersion distribution for the galaxy cluster MACSJ1206.20847. Marked are the brightest cluster galaxy (BCG) and the second brightest galaxy (GR), which is used as a reference for the Einstein radius scaling in this work. For the same galaxies, the dashed histogram gives the weighted velocity dispersion distribution. The mean deflection angle of a galaxy on all multiple images is used as the weight. As can be seen, the galaxies with lower velocity dispersions get down-weighted, meaning that they contribute on a lower level to the summed galaxies' lensing signal. The BCG has a velocity dispersion of $\sim 290 \mathrm{~km} \mathrm{~s}^{-1}$ from the best-fit scaling law. This agrees with Sand et al. (2004), who measure a stellar velocity dispersion of $\sigma \sim 250 \pm 50 \mathrm{~km} \mathrm{~s}^{-1}$ in the central $\sim 1^{\prime \prime} .5$ of the BCG.

a major impact is caused by galaxies with velocity dispersions between $100 \mathrm{~km} \mathrm{~s}^{-1}$ and $200 \mathrm{~km} \mathrm{~s}^{-1}$ (55\% of cluster galaxies light deflection for multiple images) or $250 \mathrm{~km} \mathrm{~s}^{-1}(60 \%)$.

For the galaxies, we obtain the following scaling law on a $95 \%$ c.l. basis:

$$
r_{\mathrm{t}}=31_{-14}^{+36} \mathrm{kpc}\left(\frac{\sigma}{186 \mathrm{~km} \mathrm{~s}^{-1}}\right)^{\frac{4}{3}}
$$

We translate the output of the MCMC sampling for the truncation radius of a galaxy with a $1^{\prime \prime}$ cosmology free Einstein radius into $1 \sigma$ and $2 \sigma$ confidence contours for $\sigma_{\mathrm{GR}}$ and $r_{t, \mathrm{GR}}$ and show them in Figure 9. If we were able to constrain only the mass $M\left(<R_{\text {mass,p }}\right)$ within one scale $R_{\text {mass,p }}$ (as is the case for the strong-lensing analysis of galaxies with one multiple image or one Einstein radius only), then the contours would extend to an infinite truncation radius and also smaller minimum values, as given by Equation (4):

$$
\sigma^{2}=\frac{G M\left(<R_{\text {mass }, \mathrm{p}}\right)}{\pi}\left[R_{\text {mass }, \mathrm{p}}+r_{\mathrm{t}}-\sqrt{R_{\text {mass }, \mathrm{p}}^{2}+r_{\mathrm{t}}^{2}}\right]^{-1} \text {. }
$$

Hence, the contours in Figure 9 demonstrate that the degeneracy between the two free parameters is broken (albeit not yet completely). This implies that not only the enclosed mass at some radius but also the gradient of the mass profile at this radius must be constrained by the observables, i.e., there must exist a scale $R_{\text {mass,p }}$ where the profile is best determined. In other words, there must be a location where the enclosed mass is most equal for all $\sigma_{\mathrm{GR}}$ and $r_{t}$,GR pairs of the chain output. We use Equation (4) for all MCMC sample output pairs and find this scale to be $R_{\text {mass }, \mathrm{p}}=4$.' $7 \widehat{=} 26.6 \mathrm{kpc}$. The enclosed mass at this scale becomes $M\left(<R_{\text {mass, } \mathrm{p}}\right)=7.3 \times 10^{11} M_{\odot}$ for the most likely $\sigma_{\mathrm{GR}}$ and $r_{\mathrm{t}, \mathrm{GR}}$ pair. The curve of this constant enclosed mass is added as thick dashed line in Figure 9. As expected, it traces the degeneracy in $\sigma_{\mathrm{GR}}$ and $r_{\mathrm{t}, \mathrm{GR}}$ parameter space.

We then use Equation (4) at this fixed enclosed mass radius and calculate the mass within $R_{\text {mass,p }}=4$."7 for each pair in the MCMC sample. From this distribution of enclosed masses, we take the central $68 \%$ as the error interval and obtain an enclosed mass of $M\left(<R_{\text {mass,p }}\right)=7.3 \pm 0.6 \times 10^{11} M_{\odot}$ at the fixed enclosed mass radius of $R_{\text {mass,p }}=4$.'7. These $68 \%$ upper and lower confidence values are plotted as dashed lines in Figure 9. 


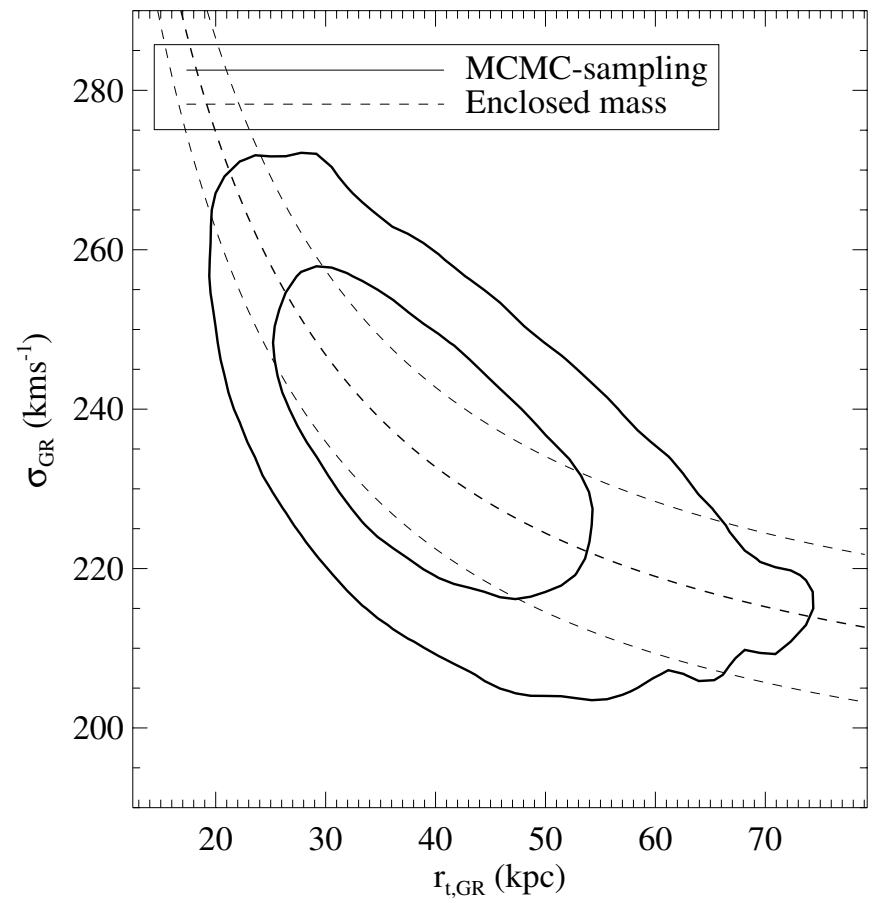

Figure 9. Probability contours for the two parameters governing the profile of the GR for the point source modeling: the truncation radius $r_{\mathrm{t}, \mathrm{GR}}=$ $r_{\mathrm{t}, 1^{\prime \prime}}\left(\sigma_{\mathrm{GR}}\left(186 \mathrm{~km} \mathrm{~s}^{-1}\right)^{-1}\right)^{1.333}$ and the velocity dispersion of the GR, $\sigma_{\mathrm{GR}}$. We also show the best fit for the enclosed mass within an effective radius as dashed lines, which gives a radius of $R_{\text {mass, }}=26.6 \mathrm{kpc}$ and an enclosed mass of $M\left(<R_{\text {mass,p }}\right)=7.3 \pm 0.6 \times 10^{11} M_{\odot}$ for the GR.

Thus, we conclude that our lens model is indeed not only sensitive to the total mass associated with galaxies but also to the size of the galaxy dark matter halos. There remains a degeneracy between the halo velocity dispersion and truncation radius at a level of a factor of two for the truncation radius. For the reference halo GR within a radius $R_{\text {mass, }}=4.7 \widehat{=} 26.6 \mathrm{kpc}$, the enclosed mass is $M\left(<R_{\text {mass, }}\right)=7.3 \pm 0.6 \times 10^{11} M_{\odot}$. For galaxies with a different luminosity and therefore a different velocity dispersion and truncation radius, the radius where the mass is best known and the mass within this radius scale like $R_{\text {mass, } \mathrm{p}} \propto r_{\mathrm{t}} / r_{\mathrm{t}, \mathrm{GR}}$ and $M\left(<R_{\text {mass }, \mathrm{p}}\right) \propto \sigma^{2} r_{\mathrm{t}} /\left(\sigma_{\mathrm{GR}}^{2} r_{\mathrm{t}, \mathrm{GR}}\right)$, respectively.

To constrain the truncation scaling even further, we need to more densely trace the lensing signal at various galaxy distances. This is achieved with the pixel-by-pixel image reconstruction of the giant arc since every pixel has a different distance to the centers of the surrounding galaxies.

\section{STRONG LENSING MODELING OF THE FULL SURFACE BRIGHTNESS OF THE GIANT ARC AND ITS COUNTERIMAGE}

We aim to further constrain the scaling relation for the truncation radius in this section. To this end, we take a different approach, reproducing the full SFB of the giant arc and its counterimage. The full SFB not only contains information about the deflection angle, but also about its derivative, making it a good tool to explore galactic halo truncation in this system.

\subsection{Setup of the Model}

We use data from the F435W, F606W, and F814W bands for the extended image reconstruction. We utilize different filters to minimize the effects of light pollution of the surrounding galaxies. The cluster galaxies are significantly dimmer in the
F435W filter, therefore minimizing the possibility of galaxy light disturbing the arc light. Since the arc is already faint in this filter (the average signal-to-noise ratio $(\mathrm{S} / \mathrm{N})$ in the mask area used is $\sim 0.5$ ), we do not consider even bluer bands. We also include a redder filter $(\mathrm{F} 606 \mathrm{~W})$ in which the arc, as well as the surrounding galaxies, becomes brighter. We add the F814W filter with an even brighter arc. In this filter, the systematic uncertainty from the subtraction of the surrounding galaxies' light is comparable to the noise in the arc region, hence we refrain from investigating even redder bands. We apply GALFIT to subtract the light of the surrounding galaxies G1 to G5; see Figure 10. For the F435W and F606W filter data, we fit a de Vaucouleurs profile (de Vaucouleurs 1948) as a light model to the data and subtract it. For these two filters, the subtracted fluxes at the position of the arc are small compared to the intrinsic noise of the images for these pixels, so the impact of the exact details of the subtracted galaxy's light model are small. This is not the case for the F814W filter, and therefore we create a best-fit de Vaucouleurs, a best-fit Sérsic (Sérsic 1963), and a best-fit King profile for galaxies G1 to G5. From these three light models, we create a mean model and subtract that from the observed image. To account for the systematic error introduced by the light subtraction in the F814W filter, we add the difference of the maximum and minimum value in each pixel for the three models to the error image derived before. We limit the analysis to a small region around the arc and its counterimage for computational reasons. This masked region is shown in Figure 10. The region is chosen by eye based on the arc visible in the F814W filter and is used in all three bands.

As a systematic test, we chose the region to be reconstructed also on the basis of an $\mathrm{S} / \mathrm{N}>2$ cut on the F814W frame. Before the modeled area was selected, the $\mathrm{S} / \mathrm{N}$ map was blocksmoothed with a length of 7 pixels. This technique led to a slightly different selection of the modeled region. However, the changes introduced into the truncation law by changing the mask are small, as described below.

For the source reconstruction, we used a $9 \times 9$ pixel grid with a free pixel scale and source plane position; the physical size of the reconstructed source is unrestricted by the number of source pixels. We compared different numbers of source pixels later on. For details of the extended SFB reconstruction, see Suyu et al. (2006) and Suyu \& Halkola (2010). Our technique uses a linear inversion method (Warren \& Dye 2003) in a Bayesian framework (Suyu et al. 2006). We searched for the most probable solution of the nonlinear lens mass parameters by maximizing the posterior in reconstructing the source (see Equation (11) of Suyu \& Halkola 2010). The lens parameter space is sampled by MCMC methods. We tried both curvature and gradient forms of regularization, and found that the resulting lens parameters were insensitive to the choice of regularization.

\subsection{Results for the Full Surface Brightness Reconstruction}

We now concentrate on modeling the galaxies G1 to G5 around the arc that are already subtracted in Figure 10. We fix all parameters (shear, cluster halo, source redshifts, and galaxy parameters) to their best-fit values from Section 5.2, and now only model galaxies G1 to G5. For the galaxies G1, G2, G4, and G5, we allowed each galaxy to have its own orientation and Einstein radius, keeping a joint truncation scaling law following Equation (14) for these galaxies. The values derived in Sections 5.1.2 and 5.2, used as starting values, are listed in Table 4. 


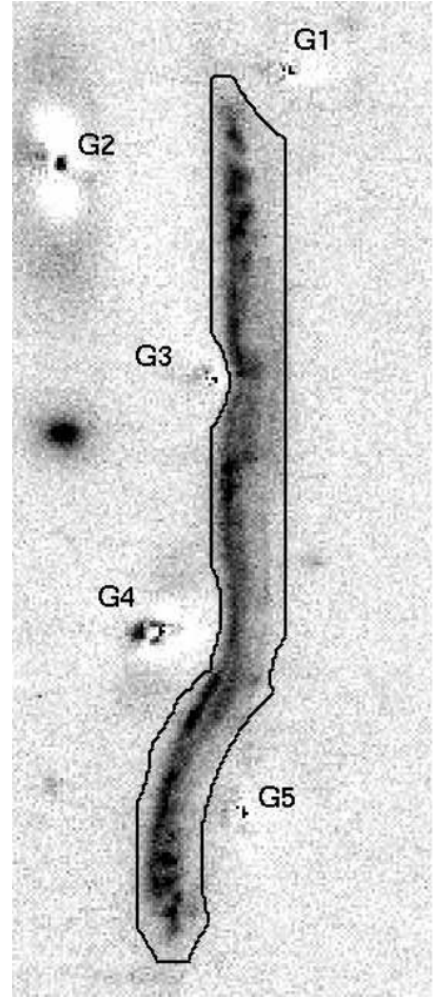

(a)

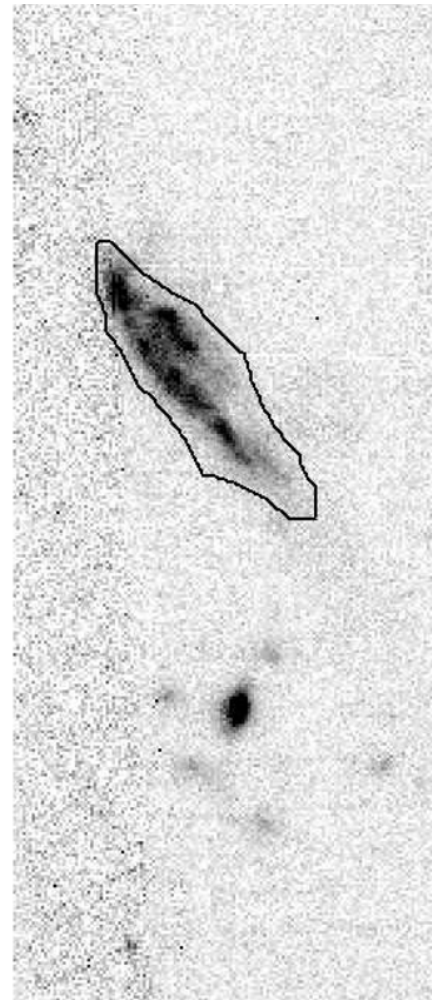

(b)
Figure 10. Frame displaying the region used around the giant arc (a) and its counterimage (b) in this galaxy cluster. The mask is outlined in black. The underlying image is the F814W observed image for this cluster. The galaxies marked with G1 to G5 in (a) have been subtracted to minimize possible contamination of the arc light from the galaxies. One pixel corresponds to 0.'05. North is up and east is to the left.

Table 4

Galaxies G1 to G5; Results from the Point-like Model in Section 5.2

\begin{tabular}{ccccccccc}
\hline \hline & $z$ & $\begin{array}{c}\Theta_{1}{ }^{\mathrm{a}} \\
\left(^{\prime \prime}\right)\end{array}$ & $\begin{array}{c}\Theta_{2}{ }^{\mathrm{a}} \\
\left(^{\prime \prime}\right)\end{array}$ & $q$ & $\begin{array}{c}\Theta_{\mathrm{pt}} \\
\left(^{\circ}\right)\end{array}$ & $\begin{array}{c}\sigma_{\mathrm{pt}} \\
\left(\mathrm{km} \mathrm{s}^{-1}\right)\end{array}$ & $\begin{array}{c}r_{\mathrm{t}, \mathrm{pt}} \\
(\mathrm{kpc})\end{array}$ & $M_{B}$ \\
\hline G1 & $0.4449^{\mathrm{b}}$ & 21.592 & 5.996 & 0.79 & 18.5 & $121_{-15}^{+16}$ & $13_{-6}^{+15}$ & -19.46 \\
G2 & $0.46 \pm 0.06^{\mathrm{c}}$ & 17.846 & 4.499 & 0.68 & -47.3 & $190_{-25}^{+26}$ & $24_{-11}^{+28}$ & -21.06 \\
G3 & $0.53 \pm 0.04^{\mathrm{c}}$ & 20.365 & 1.021 & 0.91 & -68.9 & $143_{-20}^{+19}$ & $16_{-8}^{+19}$ & $\ldots$ \\
G4 & $0.4380^{\mathrm{b}}$ & 19.473 & -3.083 & 0.80 & 25.5 & $139_{-19}^{+19}$ & $16_{-7}^{+18}$ & -19.94 \\
G5 & $0.4446^{\mathrm{b}}$ & 20.862 & -6.007 & 0.71 & -74.9 & $104_{-14}^{+14}$ & $11_{-5}^{+12}$ & -18.94 \\
\hline
\end{tabular}

Notes. The errors give $95 \%$ confidence, derived from the respective errors in Section 5.2. $M_{B}$ is calculated independently from the HST photometry, assuming a galaxy redshift of $z=0.44$.

${ }^{a}$ Relative to the center of the BCG at 12:06:12.134 R.A. (J2000), -08:48:03.35 decl. (J2000).

b Spectroscopic redshift.

c Photometric redshift estimate, $95 \%$ confidence.

We do not enforce a scaling law on G3, since it is unknown whether this object is a cluster member (it has a different photometric redshift and is formally not in our cluster member catalog). Therefore, G3 is modeled with three free parameters: its orientation, Einstein radius, and truncation radius. We obtain a best-fit model using all 12 free parameters, optimizing the F435W, F606W, and F814W filter data simultaneously. The best-fit data, model, and residuals for each of the three filters are shown in Figures 11-13.

The statistical error was estimated again using an MCMC sampling of the parameter space. The most likely values and the
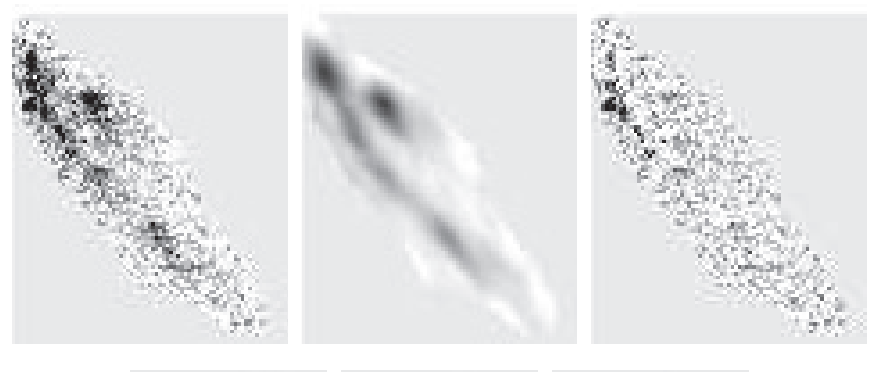

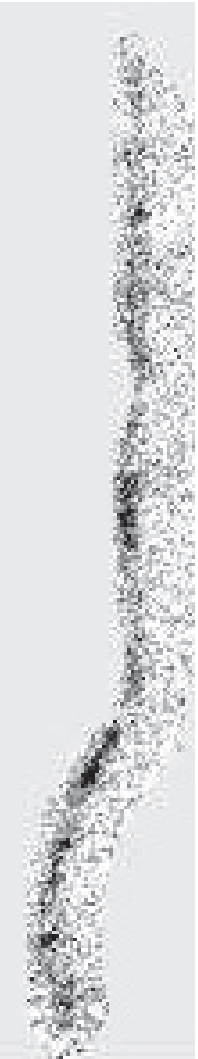

(a)

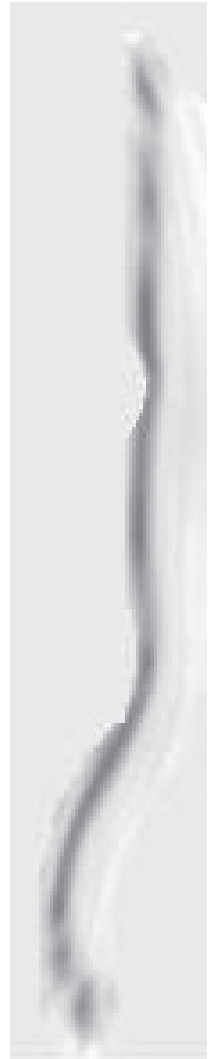

(b)

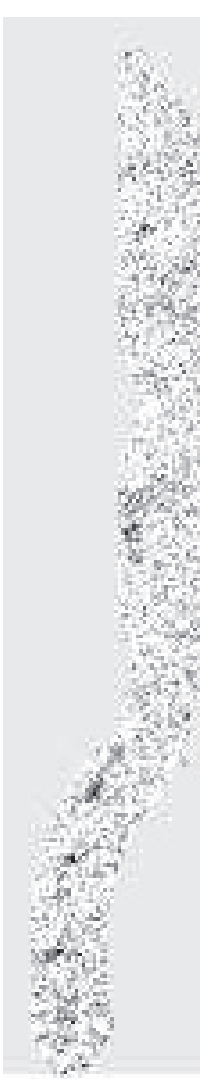

(c)
Figure 11. Arc and its counterimage reconstruction in the F435W filter. From left to right, the data, the model, and the residuals are shown. The top row shows the counterimage and the bottom row shows the giant arc. The levels of gray are the same in each image. For this figure, a source size of $20 \times 20$ pixels was used.

Table 5

Most Likely Values and Errors for the Full Surface Brightness Model of the Arc and its Counterimage

\begin{tabular}{lcccr}
\hline \hline & $\begin{array}{c}r_{\mathrm{t}, 1^{\prime \prime}} \\
(\mathrm{kpc})\end{array}$ & $\begin{array}{c}\Theta \\
\left.{ }^{\circ}\right)\end{array}$ & $\begin{array}{c}\sigma \\
\left(\mathrm{km} \mathrm{s}^{-1}\right)\end{array}$ & \multicolumn{1}{c}{$\begin{array}{c}r_{t}^{\mathrm{a}} \\
(\mathrm{kpc})\end{array}$} \\
\hline $\mathrm{G} 1$ & & $-1.5_{-3.7}^{+3.3}$ & $130_{-11}^{+10}$ & $21_{-4}^{+4}$ \\
$\mathrm{G} 2$ & & $-49.9_{-0.8}^{+0.8}$ & $165_{-2}^{+2}$ & $29_{-2}^{+2}$ \\
$\mathrm{G} 4$ & $34.2_{-1.2}^{+1.2}$ & $-1.4_{-2.3}^{+2.3}$ & $143.1_{-1.2}^{+1.2}$ & $24.1_{-1.5}^{+1.5}$ \\
$\mathrm{G} 5$ & & $-41.2_{-2.7}^{+2.5}$ & $114.9_{-1.5}^{+1.5}$ & $17.9_{-1.5}^{+1.5}$ \\
\hline
\end{tabular}

Notes. Given are the $95 \%$ c.l. errors. The best-fit cluster model from Section 5.2.1 is used as the cluster model.

${ }^{a}$ Calculated for the galaxies from the scaling law.

errors for $r_{\mathrm{t}, 1^{\prime \prime}}$ and the truncation radius for each of the galaxies can be seen in Table 5.

The truncation for the individual galaxies still follows Equation (14) with $\sigma^{\star}=186 \mathrm{~km} \mathrm{~s}^{-1}$. For every galaxy, we 


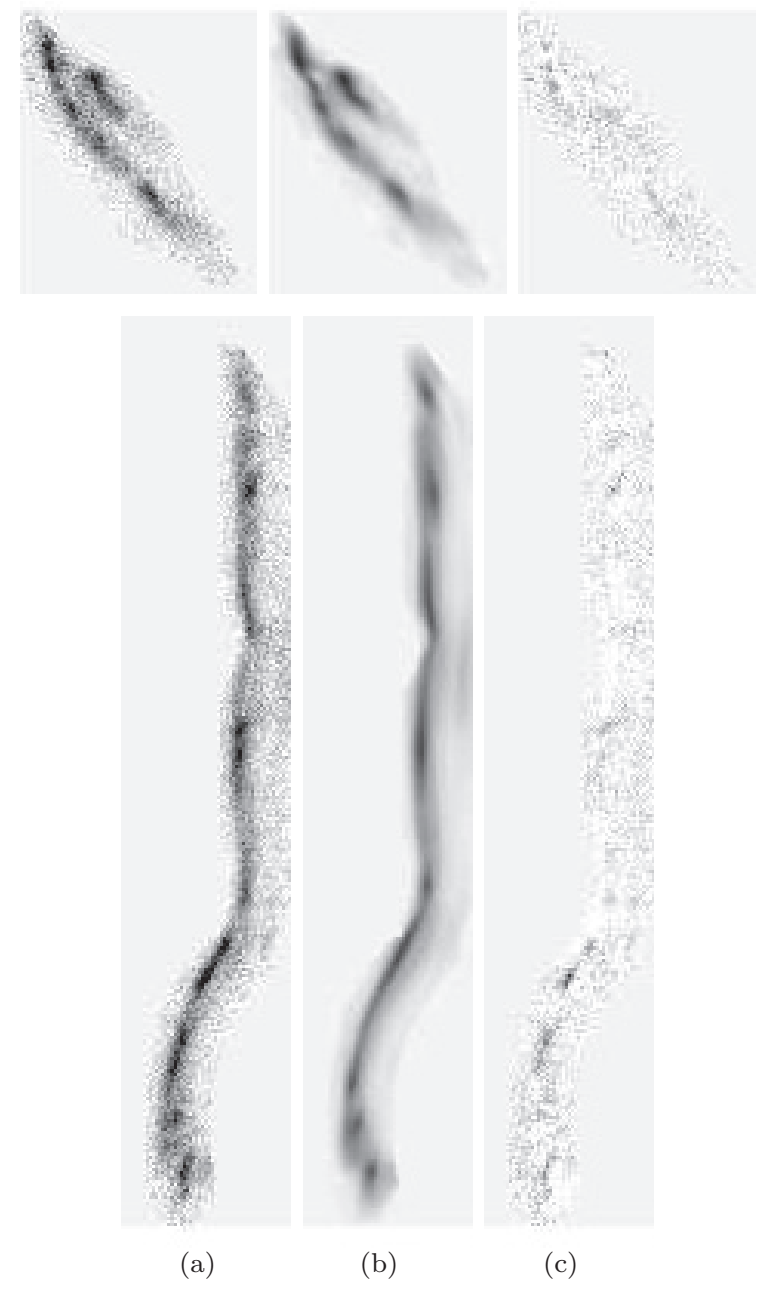

Figure 12. Same as Figure 11, this time for the F606W filter.

provide its most likely values and the $95 \%$ c.l. errors. The truncation uncertainties for each of the galaxies are derived from the uncertainties on the Einstein radii and the truncation scaling law. By comparing Tables 4 and 5, we note that the truncation scaling amplitude and the Einstein radii for the galaxies agree with each other within the errors, but that tighter constraints are recovered from the extended image reconstruction. The orientations of the galaxies in Tables 4 and 5 change by $\approx 20^{\circ}$ to $30^{\circ}$, meaning that there is a misalignment between light and total mass for these galaxies. This misalignment value is slightly higher than the $\approx 18^{\circ}$ found by Bolton et al. (2008) for isolated early-type strong-lensing galaxies.

Suyu \& Halkola (2010) quote a misalignment of their satellite light and dark matter major axis of about $50^{\circ}$. Knebe et al. (2008) show from $N$-body simulations that satellite halos as a whole prefer to be radially aligned with respect to the centers of their host halos, but not the satellites' inner parts (which predominantly trace the light distribution). This result leads to a misalignment between the light and the dark matter of satellite galaxies. Our misalignment is not as high, but nevertheless it would be worthwhile to study how tidal effects can alter the major axis of dark matter halos.

In Figure 14, the observed arc (Figure 14(a)) and its counterimage (Figure 14(b)) are shown in the left column and the top row of the middle column; the same is shown for a replacement of the arc and its counterimages with its full SFB reconstruction from its best-fit models in the left column (Figure 14(f)) and the
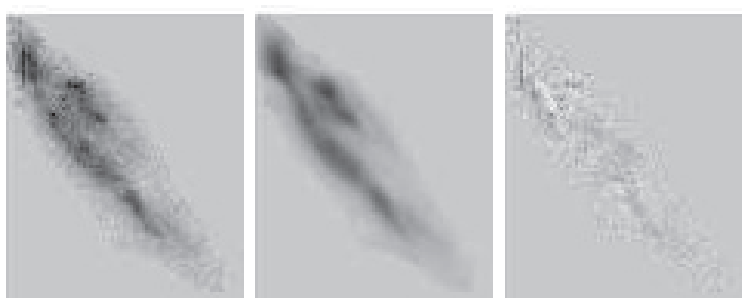

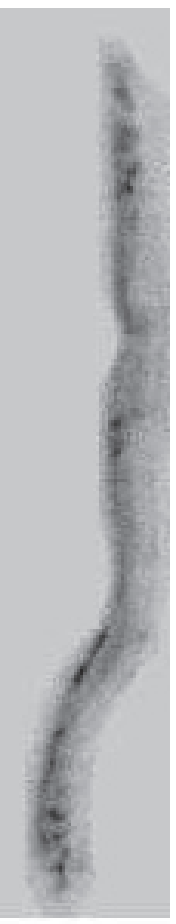

(a)

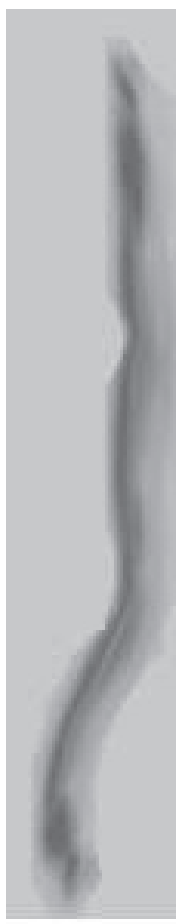

(b)

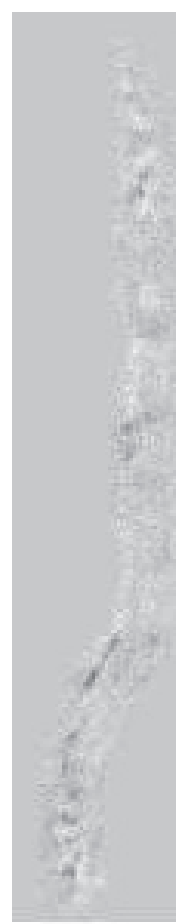

(c)
Figure 13. Same as Figure 11, this time for the F814W filter.

bottom row of the middle column (Figure 14(e)). The angular scales are given in the figures. The reconstructed source can also be seen in this figure as the two panels in the middle column (Figures 14(c) and (d)). It is fully lensed into the counterimage and only partly lensed into the arc itself. There are two versions of the source, one with $50 \times 50$ pixels, giving a resolution superior to $H S T / A C S$, and a $25 \times 25$ pixel source, showing the same source as it would be observed at approximately HST/ACS resolution. Both sources show the same field of view of 0.'94 in the $x$-direction and 1".42 in the $y$-direction, respectively. To estimate the magnification of the counterimage, we mapped the masked area in Figure 10(b) $\left(A_{\mathrm{CI}}=6.3 \mathrm{arcsec}^{2}\right)$ back into the source plane and obtained an area of $A_{\mathrm{sr}}=1.1 \operatorname{arcsec}^{2}$. Therefore, the magnification of the counterimage is $\mu_{\text {counterimage }}=5.8$. We repeated this technique with the $\mathrm{S} / \mathrm{N}$-based mask mentioned above $\left(A_{\mathrm{CI}}=5.2 \operatorname{arcsec}^{2}\right.$ and $\left.A_{\mathrm{sr}}=0.9 \operatorname{arcsec}^{2}\right)$ and obtained the same value for the magnification. Also, a direct calculation of the Jacobian matrix at the position of the counterimage provides a similar value.

Although the above statements were made for the best-fit cluster model, we then marginalized over the variety of cluster distributions compatible with the observations. To estimate the uncertainty related with the cluster model, we repeated the extended model analysis for 30 random cluster representations. These representations were taken from the MCMC sampling calculated in Section 5.2 to estimate the error. The results are presented in Table 6. 


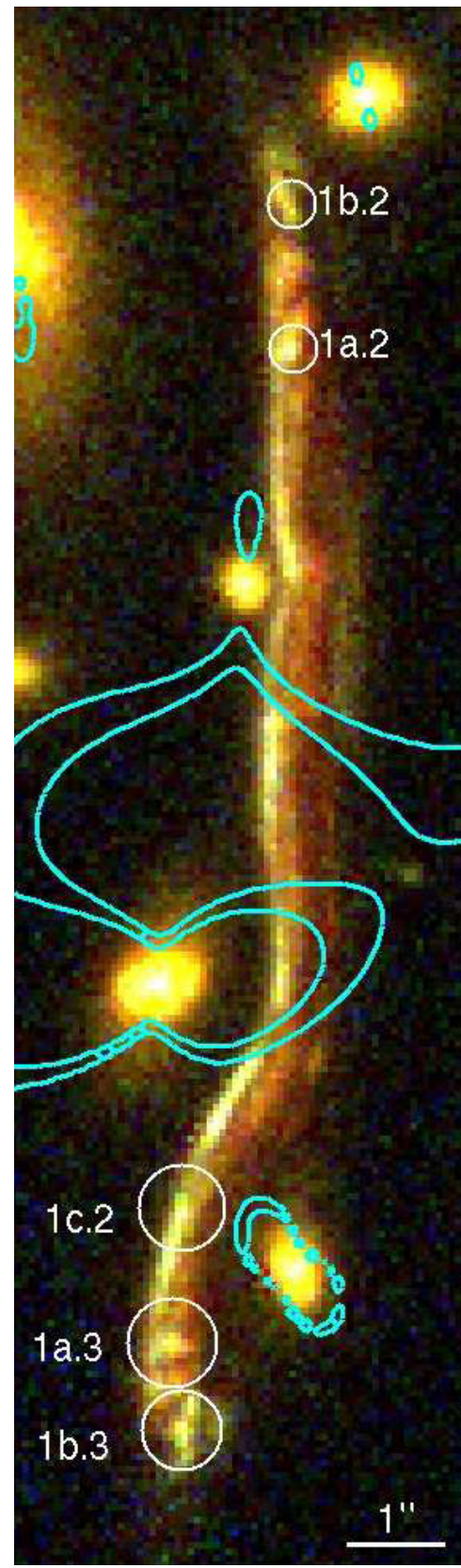

(a)

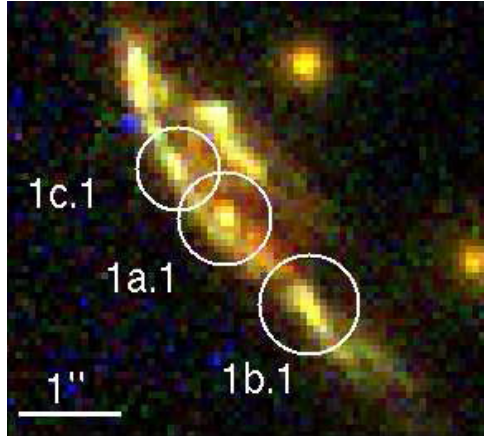

(b)

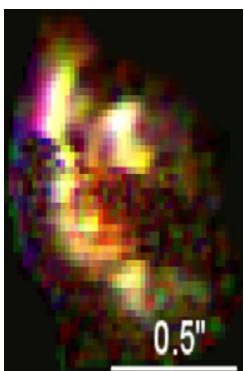

(c)

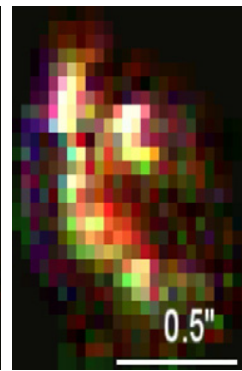

(d)

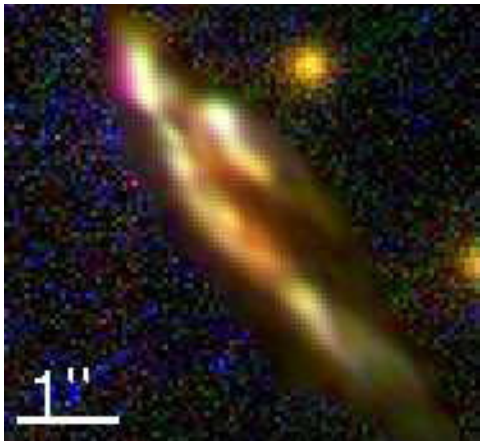

(e)

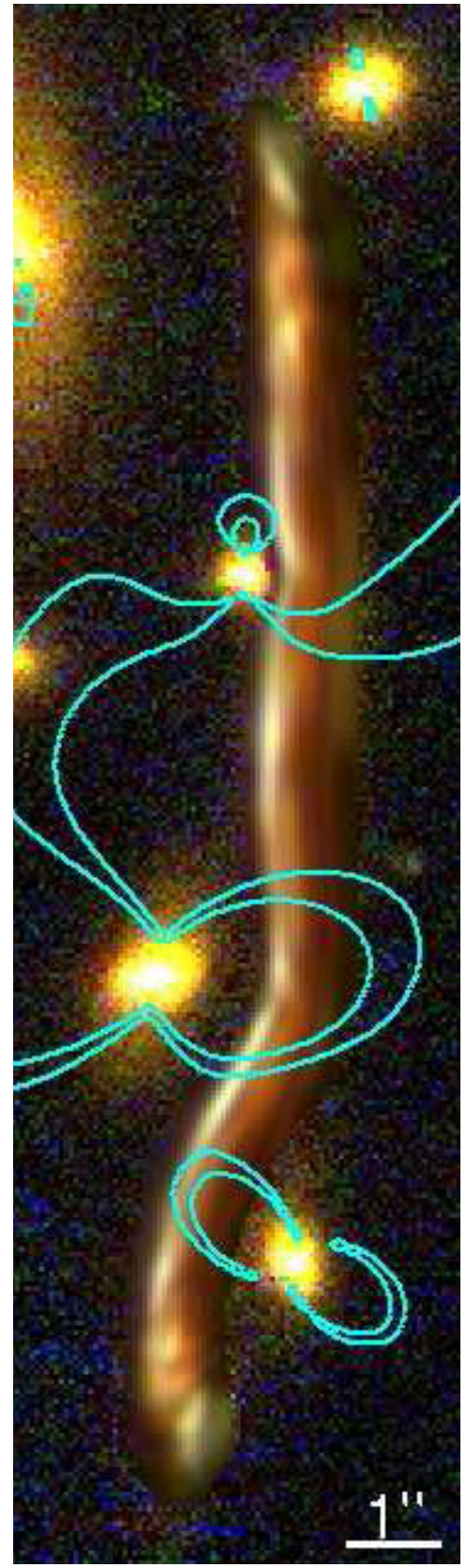

(f)

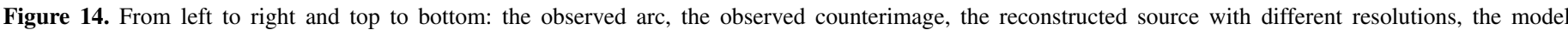

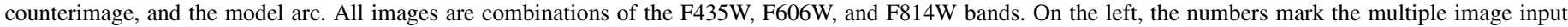

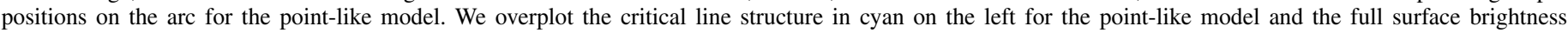

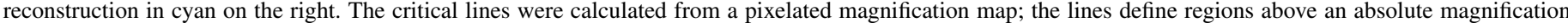

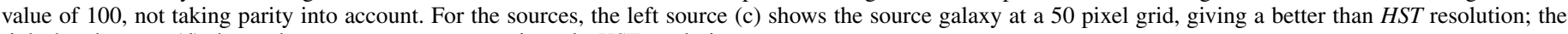
right-hand source (d) shows the same source at approximately HST resolution.

We see that the errors on the parameter estimates are increased compared to those given in Table 5 when the uncertainties from the cluster model are taken into account. For the truncation, we obtain slightly tighter constraints than the point-like model described in Equation (16) in Section 5.2. We obtain

$$
r_{\mathrm{t}}=35 \pm 8 \mathrm{kpc} \times\left(\frac{\sigma}{186 \mathrm{~km} \mathrm{~s}^{-1}}\right)^{\frac{4}{3}}
$$

The velocity dispersions and truncation radii for galaxies G1, G2, G4, and G5 for the different clusters are plotted in Figure 15.

\subsection{Tests for Systematic Errors}

The statistical error for the truncation scaling in this galaxy cluster is on the order of $25 \%$, making this method in principle a good tool for studying study truncation of galaxies. We now investigate the robustness of the truncation and Einstein radii results derived in Section 6.2 against possible sources of systematic error. Possible systematic effects might stem from the treatment of the data of the filters or the frames themselves, the analyzed arc region, the number of source pixels, or the forced scaling law. First, we repeat the analysis in each of the filters individually. The results for the different filters are summarized 
Table 6

Most Likely Values and Errors for the Full Surface Brightness Model of the Arc and its Counterimage, Taking Different Cluster Models into Account

\begin{tabular}{lcccc}
\hline \hline & $\begin{array}{c}r_{\mathrm{t}, 1^{\prime \prime}} \\
(\mathrm{kpc})\end{array}$ & $\begin{array}{c}\Theta \\
\left({ }^{\circ}\right)\end{array}$ & $\begin{array}{c}\sigma \\
\left(\mathrm{km} \mathrm{s}^{-1}\right)\end{array}$ & $\begin{array}{c}r_{t} \\
(\mathrm{kpc})\end{array}$ \\
\hline $\mathrm{G} 1$ & $1.2 \pm 20.6$ & $128 \pm 18$ & $22 \pm 7$ \\
$\mathrm{G} 2$ & & $-47.0 \pm 6.1$ & $165 \pm 6$ & $30 \pm 6$ \\
$\mathrm{G} 4$ & $35 \pm 8$ & $9.3 \pm 17.6$ & $140 \pm 6$ & $24 \pm 6$ \\
$\mathrm{G} 5$ & & $-45.3 \pm 19.1$ & $124 \pm 13$ & $20 \pm 4$ \\
\hline
\end{tabular}

Notes. From the MCMC chain used to calculate the errors in Section 5.2.1, 30 random cluster representations were taken. The analysis outlined for the best-fit cluster model was repeated for each of the random cluster models. The errors give the rms errors on the galaxy parameters and are therefore marginalized over these different cluster models.

in Table 7: all values agree with each other within the $95 \%$ c.l. intervals, implying that the SFB distribution in different filters gives consistent results regarding the halo truncation. Since the F435W band data sets have lower $\mathrm{S} / \mathrm{N}$ for the arc than the data in the two redder filters considered in this work, the best-fit parameters for the model using all three filter data simultaneously are driven by the two redder bands.

Next, we change the investigated region around the arc based on a $2 \sigma$ cut of a smoothed $\mathrm{S} / \mathrm{N}$ map in the $\mathrm{F} 814 \mathrm{~W}$ filter. We again use the data from all three filters at the same time. For the mask based on the S/N level, we obtain slightly different, but consistent, values for the truncation scale and the individual Einstein radii; see Table 7 ("mask2").

Next, we use different numbers of source pixels. For the analysis, we use only the F814W filter and the standard mask. Starting from a $8 \times 8$ pixel grid and going up to a $13 \times 13$ grid, we calculate the best fit for each model. The results are again given in Table 7 ("sr pix"). We obtain a systematic uncertainty from the source pixel size comparable to the statistical uncertainties for the best-fit cluster model when we fix the cluster potential. We verify that this is also true for much different numbers of source pixels. Using a $25 \times 25$ and $30 \times 30$ pixel grid, we obtain values consistent with the ones stated in Table 6 .

Recent spectroscopic results indicate that G3 could be a member of the galaxy cluster. Hence, we repeat the above outlined analysis including G3 as a cluster member allowing for a free central velocity dispersion and orientation, but forcing G3 to follow the same scaling law for the truncation as G1, G2, G4, and G5. Doing this, there is no change in the truncation scaling or a decrease in the error bars.

Finally, we investigate how the truncation results depend on the assumed FJ index $\delta$. We use $\delta=0.25$ instead of $\delta=0.3$, still keeping $\epsilon=0$. We restart the modeling for the point-like images, fixing the global parameters and then turn again to the extended image modeling. The corresponding truncation radii are shown in the last column of Table 7 ("FJ, $\delta=0.25$ "). Here, the truncation law is:

$$
r_{\mathrm{t}}=41.8 \mathrm{kpc} \times\left(\frac{\sigma}{186 \mathrm{~km} \mathrm{~s}^{-1}}\right)^{2} .
$$

The individual velocity dispersions and derived truncation radii, however, agree with the ones derived before within the errors; see Tables 6 and 7. This result means that there is no indication for a preferred exponent of the scaling law in this work since both scaling laws give similarly good fits.

Our tests show that the systematic errors are smaller than the ones from the uncertainty of the cluster potential, making our

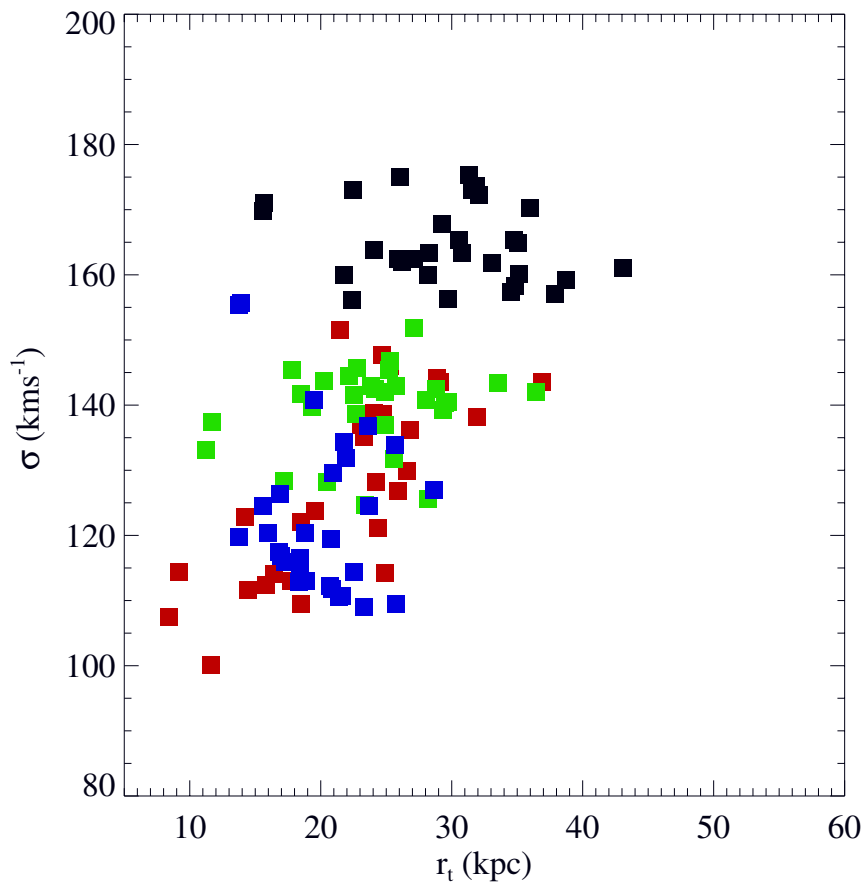

Figure 15. Velocity dispersions and calculated truncation radii for the galaxies G1, G2, G4, and G5 for the different cluster realizations. Each cluster representation has one entry for each galaxy. The symbol coding is the following: red circles: G1, black squares: G2, green stars: G4, and blue triangles: G5.

(A color version of this figure is available in the online journal.)

estimates robust with respect to systematic effects. In summary, we conclude that if we vary the weighting of the extended image input data (SFB in different filters), the masking regions, or the modeling details as the assumed FJ index, then these quantities change the estimated halo sizes less than our "statistical errors" due to different global halo models from the MCMC sample.

\section{DISCUSSION}

\subsection{Lens Modeling and Cluster Mass Distribution}

Using the positions of the multiply imaged galaxies, we measured the mass distribution in the center of MACSJ1206.20847 based on a parameterized model, where the smooth dark matter was described with an elliptical NFW profile and the matter traced by cluster galaxies was described with singular truncated isothermal ellipsoids. Using scaling relations between luminosity and velocity dispersion and between luminosity and truncation radius, the essential halo parameters (velocity dispersions and truncation radii) of all galaxies' dark matter halos were modeled with just two free parameters. The best-fit model reproduces the observed multiple image positions with a mean accuracy of $0{ }^{\prime} .85$. The level of the positional mismatch is in agreement with expectations based on unaccounted substructure or LOS contamination. For the same cluster, Zitrin et al. (2012b) obtain a slightly higher value of $\approx 1^{\prime \prime} .3$ for the average image plane reproduction uncertainty per image.

In general, the match of multiple image positions seems to depend on the number of multiple images that have been identified (Zitrin et al. 2011; Richard et al. 2010b; Limousin et al. 2008; Halkola et al. 2006). Given the number of multiple image systems, a mean image plane distance below $1^{\prime \prime}$ is a rather good value.

Finally, we find that the model would become better and require a more reasonable value for the external shear if we 
Table 7

Parameter Results for the Systematic Tests

\begin{tabular}{|c|c|c|c|c|c|c|}
\hline & $\mathrm{F} 435 \mathrm{~W}^{\mathrm{a}}$ & F606W & F814W & mask2 & sr pix ${ }^{b}$ & $\mathrm{FJ}, \delta=0.25$ \\
\hline$r_{\mathrm{t}, 1^{\prime \prime}}(\mathrm{kpc})$ & $35.1_{-4.7}^{+6.3}$ & $36.0_{-2.0}^{+2.1}$ & $36.9_{-2.0}^{+2.2}$ & 35.6 & $34.9 \pm 0.9$ & 41.8 \\
\hline$\frac{\alpha}{\delta}$ & $\frac{4}{3}$ & $\frac{4}{3}$ & $\frac{4}{3}$ & $\frac{4}{3}$ & $\frac{4}{3}$ & 2.00 \\
\hline$\sigma_{\mathrm{G} 1}\left(\mathrm{~km} \mathrm{~s}^{-1}\right)$ & $115_{-6}^{+5}$ & $126.4_{-1.4}^{+1.4}$ & $129.1_{-1.4}^{+1.3}$ & 124 & $124 \pm 8$ & 133 \\
\hline$r_{\mathrm{t}, \mathrm{G} 1}(\mathrm{kpc})$ & 18.2 & 22.6 & 21.6 & 20.1 & $20.2 \pm 1.6$ & 21.5 \\
\hline$\sigma_{\mathrm{G} 2}\left(\mathrm{~km} \mathrm{~s}^{-1}\right)$ & $161_{-7}^{+6}$ & $161_{-2}^{+2}$ & $166_{-2}^{+2}$ & 164 & $162 \pm 3$ & 165 \\
\hline$r_{\mathrm{t}, \mathrm{G} 2}(\mathrm{kpc})$ & 28.4 & 31.1 & 30.1 & 29.3 & $29.0 \pm 0.8$ & 32.5 \\
\hline$\sigma_{\mathrm{G} 4}\left(\mathrm{~km} \mathrm{~s}^{-1}\right)$ & $132_{-5}^{+4}$ & $140.7_{-2.5}^{+2.4}$ & $141.9_{-1.2}^{+1.2}$ & 139 & $140.7 \pm 1.8$ & 143 \\
\hline$r_{\mathrm{t}, \mathrm{G} 4}(\mathrm{kpc})$ & 21.9 & 25.9 & 24.6 & 23.7 & $24.1 \pm 0.6$ & 24.7 \\
\hline$\sigma_{\mathrm{G} 5}\left(\mathrm{~km} \mathrm{~s}^{-1}\right)$ & $117.9_{-1.5}^{+1.5}$ & $117.9_{-1.5}^{+1.5}$ & $113.4_{-1.5}^{+1.5}$ & 119 & $117.9 \pm 3.5$ & 116 \\
\hline$r_{\mathrm{t}, \mathrm{G} 5}(\mathrm{kpc})$ & 19.3 & 20.8 & 18.0 & 19.3 & $19.0 \pm 0.8$ & 16.2 \\
\hline
\end{tabular}

Notes. We omit errors for the truncation radii of the individual galaxies since these can be derived from the truncation law for the individual filters. We omit all errors for the mask 2 and $\mathrm{FJ}, \delta=0.25$ models since these are similar to the ones stated in Table 5.

a The errors given are the $95 \%$ c.l. on the input parameters.

b rms errors.

accounted for the intracluster light that has an almost rectangular shape and a major axis in the direction of the major cluster axis, indicating stripped stars. This offers prospects for constraining the properties (e.g., mass) of the intracluster light component. This is, however, beyond the scope of this paper.

Our total mass profile agrees with that of Zitrin et al. (2012b) and Umetsu et al. (2012). Regarding values for concentration and scale radius for the total cluster mass distribution, we refer the reader to the work of Umetsu et al. (2012) as the mass profile in that work was constrained on much larger scales using strongand weak-lensing shear and magnification information.

In addition to previous work, we pay special attention to match the extended SFB distribution of the giant arc and its counterimage as observed in the F435W, F606W, and F814W filters. This helps us to constrain the velocity dispersion and truncation parameters of cluster galaxy halos considerably beyond the result obtained from our point source modeling alone. We ensured that the results were robust regarding modeling details and the exact information used from the extended light distribution of the arc.

\subsection{Halo Velocity Dispersion versus the Faber-Jackson Relation}

The amplitudes for the luminosity versus velocity dispersion scaling law (and the luminosity versus truncation radius scaling law) were constrained without any reference to optical galaxy properties. We obtained the following relation between the apparent $\mathrm{AB}$ magnitude in the F160W filter and the halo velocity dispersion:

$$
m_{160, \mathrm{AB}}=-8.333 \log \left(\sigma\left[\mathrm{km} \mathrm{s}^{-1}\right]\right)+37.39 .
$$

In the above relation, the value for the slope was assumed and the zero point was determined. The lensing-derived velocity dispersion in this work agrees with the measured stellar velocity dispersion for the BCG. Recent measurements also indicate an agreement between the lensing-derived and measured velocity dispersions for the GR.

It is known from field elliptical strong lenses that multiple image systems can be well reproduced assuming an isothermal total mass profile with an amplitude given by the central stellar velocity dispersion. This isothermality is measured out to two Einstein radii (Koopmans et al. 2006; Grillo et al. 2010; Eichner et al. 2012). However, since Einstein radii of elliptical galaxies are typically of the order of the effective radius, the mass distribution is only measured out to one effective radius with strong lensing of field elliptical galaxies. This is the scale where the stellar mass is still dominating or at most the dark matter and luminous matter are of the same order. We wish to compare the lensing-derived FJ relation from this work with a local estimate from Kormendy \& Bender (2013). For this comparison, we need the absolute $B$-band magnitudes $M_{B}$ for the cluster members evolved to $z=0$. For all galaxies in our cluster member catalog, we fit the spectral energy distribution (SED) using their full 16 filter photometry (see Figure 10 of Postman et al. 2012), assuming that all the galaxies are at $z=0.44$. In this way, we obtained for each cluster member its SED type and an estimate for its rest-frame absolute magnitude in the Bessel $B$ band, $M_{B}$ (in the Vega system). We then applied redshift evolution to the elliptical galaxies' FP mass-to-light ratios, where we assumed that the evolution was due to the aging of the stellar population (luminosity evolution). Saglia et al. (2010) measured this in the EDISC sample with cluster (and field) elliptical galaxies and obtained an evolution of the mass-to-light ratio of cluster elliptical galaxies of $\Delta \log M / L_{B}=-1.6 *(1+z)$, which corresponds to a flux dimming factor of 1.8 from $z=0.44$ to $z=0$. We plot the luminosity-evolved absolute $B$-band magnitudes of red cluster members versus their halo velocity dispersions in Figure 16. The velocity dispersion results for the $\delta=0.3$ case are shown in yellow and those for the $\delta=0.25$ case are shown in red. We do not change the halo velocity dispersion when evolving the cluster elliptical galaxies to redshift zero, since at fixed stellar mass there is hardly any evolution of the stellar velocity dispersion from $z=0.44$ to $z=$ 0 according to Figure 22 of Saglia et al. (2010). We assumed that the same also held true for the halo velocity dispersion. We also drew errors of $10 \%$ on the velocity dispersions in Figure 16 to guide the eye, since this is the accuracy at which we can determine the amplitude of the luminosity versus velocity dispersion scaling. In the same figure, we added the local FJ relation from Kormendy \& Bender (2013) as a green line. Its slope (in our notation) is $\delta_{\mathrm{FJ}}=0.273$ and thus in between our assumed $\delta=0.25$ (red triangles) and $\delta=0.3$ (yellow circles) cases. Both results agree within their errors with the FJ relation, although the $\delta=0.3$ case is shifted to lower velocity dispersions at the faint end. 


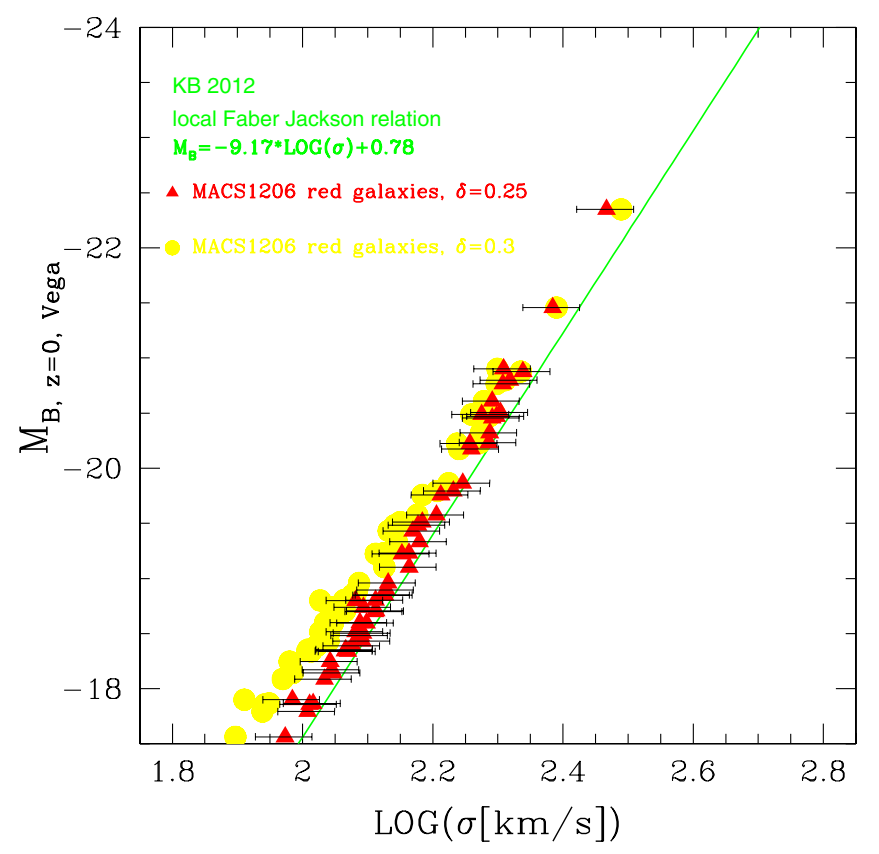

Figure 16. Local Faber-Jackson relation in absolute Vega $B$ magnitudes vs. the central stellar velocity dispersion from Kormendy \& Bender (2013) (shown with a line). The triangles show the absolute $B$ magnitude of MACSJ1206.2 red cluster members corrected for luminosity evolution to redshift zero by a factor of 1.8 vs. the halo velocity dispersion obtained from the lens modeling. Note that we do not model each galaxy separately but only the amplitude of the relation for the assumed scaling law (in this case, $\delta=0.25$ ). The filled circles show the same galaxies for the assumed scaling law of $\delta=0.30$. The scatter around the $\delta=0.25$ slope is due to the fact that the luminosity- $\sigma$ scaling was applied using the NIR F160W data and not the rest-frame $B$ magnitude obtained from the SED fitting. The small scatter demonstrates that the SEDs of the red galaxies are fairly uniform.

(A color version of this figure is available in the online journal.)

Up until now, we assumed the stellar and halo velocity dispersions to be equal. In the following, we want to address the possible differences between stellar velocity dispersion and the dark matter halo velocity dispersion. We have shown in Section 5.2 that we constrain the mass profile of our cluster galaxies most strongly at a scale of $\sim 5$ effective radii. This position is where dark matter dominates and thus we now can compare the halo velocity dispersion derived from lensing with the stellar velocity dispersion amplitude. An estimate for the stellar velocity dispersion amplitude can be obtained from either the FJ (Faber \& Jackson 1976) or the FP relation (Bender et al. 1992). Stars in elliptical galaxies are dynamically colder than their dark matter halo (see Gerhard et al. 2001) and their velocity dispersion is linked to the maximum circular halo velocity as $\sigma_{\text {stars }}=0.66 v_{\text {circ }}^{\max }$ (at least for the sample of elliptical galaxies investigated in Gerhard et al. (2001); see their Equation (2)). Therefore, we would expect the halo velocity dispersion to be $\sigma_{\text {halo }}=1.07 \sigma_{\text {stars }}$. Our best-fit halo velocity dispersions in Figure 16 are slightly smaller than those of the stars according to the $\mathrm{FJ}$ relation derived in Kormendy \& Bender (2013), but considering the uncertainty on the measured halo velocity dispersion, this result is not significant. We would need a more precise global cluster model (to decrease the error on the halo velocity dispersions) and spectroscopic stellar velocity dispersions for the red cluster members to measure the relation between halo and stellar velocity dispersion more precisely.

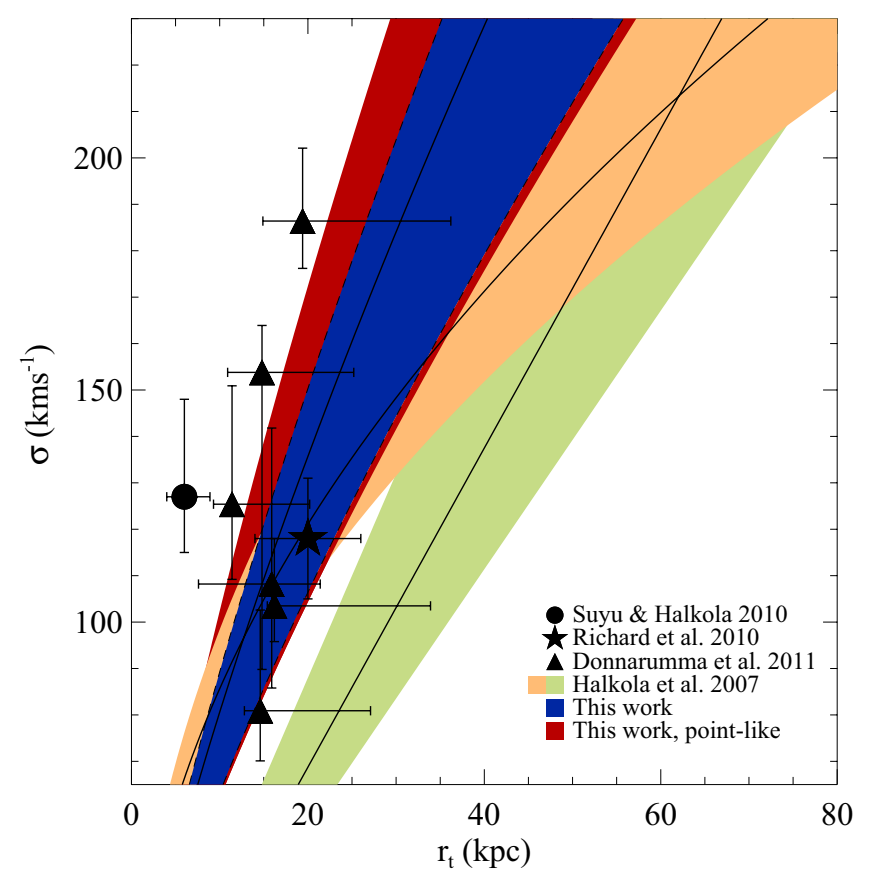

Figure 17. Our results for the halo truncation radius vs. velocity dispersion for the point source modeling (the region marks the $68 \%$ confidence interval) and the SFB modeling of the arc (the best fit is the black line, and the $1 \sigma$ confidence region). The triangles mark constraints (and their $1 \sigma$ errors) for individual galaxies obtained by Donnarumma et al. (2011), the star marks the result for one galaxy from Richard et al. (2010a), and the point is taken from Suyu \& Halkola (2010). The other areas mark the $1 \sigma$ confidence intervals obtained from Halkola et al. (2007) for two different scaling relations, $r_{\mathrm{t}} \sim \sigma$ and $r_{\mathrm{t}} \sim \sigma^{2}$, as analyzed in their work.

(A color version of this figure is available in the online journal.)

\subsection{Halo Truncation and Stripped Mass Fraction}

The truncation radius versus velocity dispersion relation for the halo of cluster members is:

$$
r_{\mathrm{t}}=(35 \pm 8 \mathrm{kpc})\left(\frac{\sigma}{186 \mathrm{~km} \mathrm{~s}^{-1}}\right)^{\frac{4}{3}},
$$

from the full SFB reconstruction of the extended arc and its counterimage, based on four nearby cluster galaxies. We obtain a very similar relation for the point-like modeling, which includes all cluster members statistically. We have shown in Figure 8 that the galaxies contributing most strongly to our point-like halo truncation measurement have velocity dispersions between $100 \mathrm{~km} \mathrm{~s}^{-1}$ and $200 \mathrm{~km} \mathrm{~s}^{-1}$. In Equation (19), the exponent 4/3 is assumed to be known and the amplitude is determined. As can be seen in Figure 17, the errors on this relation in the range of $100 \mathrm{~km} \mathrm{~s}^{-1}$ and $200 \mathrm{~km} \mathrm{~s}^{-1}$ are quite large, hence different exponents for the truncation versus velocity dispersion law fit the multiple image positions equally well, as long as similar values for the actual truncation radii of the most relevant individual galaxies are predicted. If the exponent was changed to 2 , the results would still be very similar for the majority of galaxies and we would obtain a similar fit quality. Our velocity dispersion versus truncation radius relation is shown in Figure 17, where the error intervals obtained from the point-like modeling are in red and the errors for the extended SFB modeling are in blue.

Since the halo velocity dispersion is not a direct observable, a more practical relation than Equation (19) is to rephrase Equation (19) as a function of apparent $m_{\mathrm{AB}, 160}$ magnitude:

$$
\log r_{\mathrm{t}}(\mathrm{kpc})=\log (35 \pm 8)-0.16 m_{160, \mathrm{AB}}+2.96,
$$


such that this equation provides a recipe to model the galaxy halos of other clusters at the same redshift. To obtain a redshift independent relation, we transform Equation (20) to relate the truncation radius of each galaxy directly to its absolute $B$-band magnitude (in Vega magnitudes). We obtain

$$
\log r_{\mathrm{t}}(\mathrm{kpc})=\log (35 \pm 8)-0.16 M_{\mathrm{B}, \mathrm{Vega}}-3.372 .
$$

This equation holds for the red galaxies in Figure 1. We now compare our results with previous work on the truncation of galaxies halos in clusters of galaxies. Halkola et al. (2007) completed a statistical analysis of all galaxies in the stronglensing regime in the cluster A1689. Although these authors included galaxies in the modeling with (FP and FJ) velocity dispersion estimates ranging from about $300 \mathrm{~km} \mathrm{~s}^{-1}$ down to about $20 \mathrm{~km} \mathrm{~s}^{-1}$ (see Figure 5 of Halkola et al. 2006), in their sample it seems that their sensitivity to halo truncation is mostly due to massive galaxies with a velocity dispersion of $220 \mathrm{~km} \mathrm{~s}^{-1}$. This effect can be seen in Figure 17, which shows that the halo truncation size for the two parameterizations $\left(s \propto \sigma\right.$ and $\left.s \propto \sigma^{2}\right)$ agrees for $\sigma=220 \mathrm{~km} \mathrm{~s}^{-1}$ galaxies where the halo size then is equal to about $65 \mathrm{kpc}$ with a $1 \sigma$ error of about $15 \mathrm{kpc}-20 \mathrm{kpc}$. Additionally, Figure 1 of Halkola et al. (2007) shows that $\chi^{2}$ starts to rise steeply only for halo sizes smaller than $30 \mathrm{kpc}$. This finding implies that their result is in agreement with ours.

The works of Richard et al. (2010a) and Donnarumma et al. (2011) allow a more direct comparison to our results since these authors analyze a situation more similar to ours. Their cluster galaxies have mostly low velocity dispersions (triangle and stars in Figure 17) and they typically have a projected distance to the cluster center of the order of $\approx 10^{\prime \prime}$.

Our median "lensing-weighted" cluster galaxy distance to the cluster center is $\sim 26^{\prime \prime}$ (the four cluster members close to the arc have a distance of $\sim 20^{\prime \prime}$, which is $6 \%$ of the virial radius of this cluster; Umetsu et al. 2012). This means that our galaxy sample and that of Richard et al. (2010a) and Donnarumma et al. (2011) are likely to have undergone a similar amount of stripping (assuming that the central cluster density and the collapse state of their clusters are similar to ours). The results of Richard et al. (2010a) and Donnarumma et al. (2011) are inserted into Figure 17 and are in agreement with ours.

Suyu \& Halkola (2010) measured the individual truncation of a satellite halo embedded in a group (for which we estimate a velocity dispersion of about $400 \mathrm{~km} \mathrm{~s}^{-1}-500 \mathrm{~km} \mathrm{~s}^{-1}$ based on their lensing model), where the satellite was only $\sim 26 \mathrm{kpc}$ away in projection from the group center. They estimated the velocity dispersion of the satellite galaxy to be around $120 \mathrm{~km} \mathrm{~s}^{-1}$ and obtained a truncation radius of only $4-9 \mathrm{kpc}$ at $95 \%$ confidence. Their result shows that indeed halo truncation can be severe close to centers of groups (and thus even more for clusters).

Using a different method, Limousin et al. (2007a) measured the truncation of cluster galaxies with weak lensing for five different clusters and obtained similar results within the errors. $\mathrm{Pu}$ et al. (2010) investigated three nearby group members using dynamical modeling. They used a common cutoff radius for all three galaxies with velocity dispersions between $\sigma \approx$ $200 \mathrm{~km} \mathrm{~s}^{-1}$ and $\sigma \approx 300 \mathrm{~km} \mathrm{~s}^{-1}$, somewhat higher than our sample. Their best-fit value is $R_{c}=60 \mathrm{kpc}$, which would agree with our measurement if we extrapolated to higher velocity dispersions.

We compare our value for the truncation radius with the halfmass radius derived in Limousin et al. (2009) from simulations of halo stripping in two numerically simulated clusters, one with a similar virial mass to MACSJ1206.2-0847. Our galaxy
G4 in Table 6 has a truncation radius of $24 \pm 6 \mathrm{kpc}$ and a $R$-band rest-frame luminosity of $L_{\mathrm{R}, \mathrm{rf}} \approx 3 * 10^{11} L_{\mathrm{R}, \odot}$. At this luminosity, Limousin et al. (2009) obtain a half-mass radius of $r_{1 / 2} \approx 20 \mathrm{kpc}$ for a galaxy close to the cluster center in projection, which agrees well with our result.

We can infer the amount of stripped dark matter for cluster galaxies if we compare their truncation radii with the truncation radii of the corresponding galaxies in the field. Brimioulle et al. (2013) measure a truncation radius of $s=245_{-52}^{+64} h_{100}^{-1} \mathrm{kpc}$ for a reference galaxy with $\sigma=144 \mathrm{~km} \mathrm{~s}^{-1}$, with a red SED and in an underdense environment. For the same velocity dispersion, our cluster galaxies have a truncation radius of $r_{\mathrm{t}}=25 \pm 6 \mathrm{kpc}$. Consequently, the ratio of the total halo mass in the field to that in the cluster for this kind of galaxy is $M_{\text {tot,field }} / M_{\text {tot,cluster }}=13.9_{-4.4}^{+4.9}$. In the last step, we have assumed that the velocity dispersion of a halo (i.e., the kinematics of stars and central dark matter particles) does not change when it is stripped during cluster infall. Models of massive galaxies ( $\mathrm{Pu}$ et al. 2010) indeed suggest that a change in the halo truncation radius (as long as it happens beyond $\sim 5 R_{\text {eff }}$ ) has no detectable influence on the stellar kinematics inside $\sim 5 R_{\text {eff }}(\mathrm{J}$. Thomas 2013, private communication). The truncation radius for the GR is $\sim 5$ times higher than the effective radius of this galaxy. Romanishin (1986) give a relation for the absolute $B$-band magnitude $M_{B} \sim-2.06 \log R_{\text {eff }}$. This means that $R_{\text {eff }}$ drops faster with fainter $M_{B}$ than $r_{\mathrm{t}}$ in Equation (21), implying that $r_{\mathrm{t}} / R_{\mathrm{eff}}$ rises for smaller fluxes and hence stripping of the galaxies does also not affect the kinematics of the lower luminosity galaxies.

The large mass loss of the cluster galaxies (close in projection to the cluster center) agrees with results from numerical modeling of the stripping (see also the Introduction), which shows that mass losses up to $90 \%$ are common for cluster galaxies close to the cluster center (Warnick et al. 2008).

If we assume that all cluster galaxies considered in our model have halo masses of only $10 \%$ of their infall mass, then the total stripped mass amounts to $M_{\text {stripped }}=5.1_{-1.5}^{+1.8} \times 10^{13} M_{\odot}$ out to a projected radius of $\approx 100 \mathrm{kpc}$. The total mass estimate at the same radius is $7.11_{-0.03}^{+0.04} \times 10^{13} M_{\odot}$. Within a projected radius of $\approx 400 \mathrm{kpc}$, the ratio of stripped to total cluster mass has values of $25 \%-50 \%$. This will be an upper value, since the fractional stripped galaxy halo masses will be smaller in the outskirts. Nevertheless, this result implies that a significant fraction of the smooth dark matter component in the cluster core originates from cluster members stripped during the formation and relaxation of the cluster.

\subsection{The SFB Distribution of the Source of the Giant arc}

Since not all of the arc source is lensed into the giant arc-basically, all parts above image 1c.1 on the counterimage are outside of the caustic and therefore only imaged one time in the counterimage and not in the arc-only the observed counterimage can be used to obtain the source properties. The observed counterimage and the best-fit source model can be seen in Figure 14, both at HST resolution and at better than $H S T$ resolution. Comparing the observed counterimage and the source at HST resolution, the increase in the level of detail due to lensing in this case can be seen. The observed counterimage (Figure 14(b)) and the high resolution delensed counterimage (Figure 14(c)) reveal the magnification of the source due to lensing. The magnification is approximately equal to $\sim 5.8$; this value corresponds to a flux brightening by about 2 mag. 
Table 8

Apparent Magnitudes of the Counterimage (CI) and Modeled Source (SR) in AB Magnitudes

\begin{tabular}{lcccccccccrrr}
\hline \hline Filter & F435W & F475W & F606W & F625W & F775W & F814W & F850LP & F105W & F110W & F125W & F140W & F160W \\
\hline CI & 22.20 & 22.14 & 21.73 & 21.52 & 20.92 & 20.76 & 20.39 & 20.25 & 20.06 & 19.93 & 19.81 & 19.72 \\
SR & 24.11 & 24.05 & 23.64 & 23.43 & 22.83 & 22.67 & 22.30 & 22.16 & 21.97 & 21.84 & 21.72 & 21.63 \\
\hline
\end{tabular}

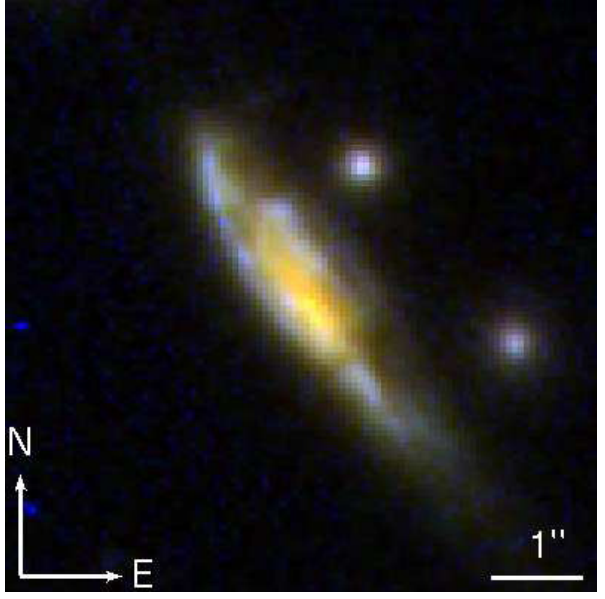

(a)

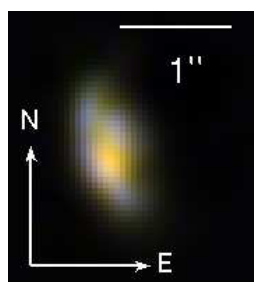

(b)

Figure 18. False color images using the F775W, F125W, and F160W frames, corresponding to approximate BRI rest-frame colors. The left image is the HST observation of the counterimage and the right image is the unlensed source as it would be observed with a pixel size of the HST. The unlensed source is convolved with a Gaussian function in each filter representing the approximate point-spread function. In the source plane, $1^{\prime \prime}$ corresponds to $8.13 \mathrm{kpc}$. We gain an increase in spatial resolution of $\sim \sqrt{5.8}$ by the gravitational telescope.

(A color version of this figure is available in the online journal.)

A three color representation of the counterimage in the F775W, F125W, and F160W filters and an approximately delensed version of it are shown in Figure 18. The filters are chosen to be equal to the rest-frame $B-, R$-, and $I$-band filters. The color image suggests that the source is a fairly inclined, spiral star-forming galaxy with a core hosting more evolved stars. Comparing with CANDLES results (Figure 2 of Wuyts et al. 2012), we conclude that the lensed galaxy is a fairly normal $z \sim 1$ galaxy. Results of the 3D HST project indicate that about half of $1<z<1.5$ galaxies have $\mathrm{H} \alpha$ emission features with rest-frame equivalent widths between $10 \AA-130 \AA$ (van Dokkum et al. 2011) and that star formation occurs inside out with $\mathrm{H} \alpha$ emission lines in the outskirts of galaxies and continuum emission from their centers (Nelson et al. 2012). Thus, it is likely that our source has emission lines, too. This makes the galaxy an ideal target for measuring the 2D kinematics with the ground-based near-infrared (NIR) integral field unit KMOS at the VLT.

In Table 8, the magnitudes of the counterimage and the source are listed. The increase in brightness due to the lensing effect makes this galaxy at $z=1.036$ much easier to observe than the unlensed source would be.

\section{SUMMARY AND CONCLUSIONS}

In this work, we measured the sizes of galaxies in the center of the galaxy cluster MACSJ1206.2-0847 using strong lensing. Measurements of the dark matter halo sizes of distant galaxies are rare, since dynamical methods are not yet sensitive enough to obtain these data. Hence, we rely on the gravitational lensing signal to study the truncation of elliptical galaxies in

a galaxy cluster. We first build a model for the cluster mass distribution based on the 12 multiple image systems with 52 multiple images stated in Zitrin et al. (2012b). We model the cluster galaxies employing scaling laws based on the NIR fluxes. We then derived the average truncation of the galaxy halos by optimization of the normalization of these scaling laws. Based on this methodology, we reconstructed the full SFB distribution of the giant arc and its counterimage in this cluster by modeling the truncation of the cluster galaxies surrounding the arc separately, obtaining consistent results for both approaches. In detail, our results are as follows.

1. We obtain a mean distance of the model predicted multiple image positions from their input positions of $\sim 0^{\prime \prime} 85$.

2. We measure a mass of $M_{\text {tot }} \sim 7 \times 10^{13} M_{\odot}$ within a (cylindrical) radius of $100 \mathrm{kpc}$, which is in good agreement with other studies of this cluster.

3. We model the individual galaxies assuming scaling relations with the F160W band flux of each galaxy, using the normalizations of these scaling laws as free parameters. We refer these normalizations to one reference galaxy and calculate values of $r_{\mathrm{t}, \mathrm{GR}}=41_{-18}^{+34} \mathrm{kpc}$ and $\sigma_{\mathrm{GR}}=236_{-32}^{+29} \mathrm{~km} \mathrm{~s}^{-1}$ for the reference galaxy. We constrain the mass distribution of cluster galaxies best at $\sim 5$ effective radii. Assuming passive luminosity evolution for the absolute $B$-band luminosity of the cluster galaxies, we show that our lensing-derived velocity dispersions agree well with values given in Kormendy \& Bender (2013) for local elliptical galaxies.

4. We reconstruct the full SFB of the giant arc and its counterimage by individually modeling the four cluster galaxies closest to it. For these four galaxies, we calculate values for the individual velocity dispersions that agree with those derived from the scaling relations. The derived sizes of the four galaxies are similar to the sizes derived from the point-like lensing model. We derive the following truncation law for cluster members when reconstructing the full SFB distribution of the arc:

$$
r_{\mathrm{t}}=(35 \pm 8) \mathrm{kpc}\left(\frac{\sigma}{186 \mathrm{~km} \mathrm{~s}^{-1}}\right)^{\frac{4}{3}} .
$$

This truncation law agrees with predictions from simulations and with other measurements carried out in dense environments. Testing different exponents of the truncation law gives consistent results for the sizes of the individual galaxies within the errors, meaning that we cannot constrain the exponent of the scaling law.

5. The truncation law stated above means that a large fraction of the dark matter halos of the cluster galaxies in this cluster have been stripped from their host galaxies as compared to field galaxies of the same velocity dispersion. Again, this result agrees with expectations from simulations.

In summary, the investigated galaxies in MACSJ1206.20847 have shrunk significantly, which is consistently derived from both point-like modeling of all multiple image systems 
and from modeling the full SFB of the arc and its counterimage. The results for the sizes of the galaxies in the center of this cluster at $z=0.44$ agree with results derived for other clusters at lower redshifts, e.g., A1689 $(z=0.183)$ or the Coma cluster, indicating that most of the truncation of galaxies close to the cluster center has already been completed for MACSJ1206.2-0847 at $z=0.44$.

The analysis presented here can be extended to other clusters in the CLASH survey, e.g., MACSJ1149.6+2223 and A383, leading to a more complete picture of galaxy sizes in dense environments and-closely connected-their relation with the cluster-scale dark matter halo.

This work is supported by the Transregional Collaborative Research Centre TRR 33-The Dark Universe and the DFG cluster of excellence "Origin and Structure of the Universe." We thank Jens Thomas, Ralf Bender, and Roberto P. Saglia for fruitful discussions of the properties of early-type galaxies. We thank the anonymous referee for his/her comments and suggestions to improve the text. Based on observations made with the NASA/ESA Hubble Space Telescope, obtained from the data archive at the Space Telescope Science Institute. STScI is operated by the Association of Universities for Research in Astronomy, Inc. under NASA contract NAS 5-26555. The CLASH Multi-Cycle Treasury Program (GO-12065) is based on observations made with the NASA/ESA Hubble Space Telescope. The Space Telescope Science Institute is operated by the Association of Universities for Research in Astronomy, Inc. under NASA contract NAS 5-26555. Part of this work is based on data collected at the Very Large Telescope at the ESO Paranal Observatory, under Program ID 186.A-0798. K.U. acknowledges partial support from the National Science Council of Taiwan grant NSC100-2112-M-001-008-MY3 and from the Academia Sinica Career Development Award. A.Z. is supported by contract research "Internationale Spitzenforschung II-1" of the Baden Württemberg Stiftung. The Dark Cosmology Centre is funded by the DNRF.

\section{APPENDIX \\ GALAXY LENSES LIST}

In this appendix, we present the list of derived galaxy lenses used for the strong-lensing model in Table 9. We show the position relative to the BCG, the ellipticity and orientation, and the best-fit Einstein and truncation radii from the best-fit model

Table 9

Derived Galaxy Lenses

\begin{tabular}{lrlrrr}
\hline \hline $\begin{array}{l}x^{\mathrm{a}} \\
\left({ }^{\prime \prime}\right)\end{array}$ & \multicolumn{1}{c}{$\begin{array}{c}y^{\mathrm{a}} \\
\left({ }^{\prime \prime}\right)\end{array}$} & $q$ & \multicolumn{1}{c}{$\begin{array}{r}\Theta_{q} \\
\left({ }^{\circ}\right)\end{array}$} & $\begin{array}{r}\sigma \\
\left(\mathrm{km} \mathrm{s}^{-1}\right)\end{array}$ & $\begin{array}{r}r_{\mathrm{t}} \\
(\mathrm{kpc})\end{array}$ \\
\hline 0.000 & 0.000 & 0.59 & 14.6 & 296.3 & 57.8 \\
-51.961 & -18.520 & 0.85 & 28.2 & 235.7 & 42.6 \\
52.028 & -23.567 & 0.87 & -47.1 & 196.6 & 33.4 \\
70.454 & -24.260 & 0.42 & -78.3 & 191.3 & 32.2 \\
79.369 & -16.726 & 0.49 & 62.4 & 188.9 & 31.7 \\
-75.133 & -19.806 & 0.56 & 36.5 & 185.7 & 31.0 \\
17.846 & 4.499 & 0.68 & -47.3 & 182.3 & 30.2 \\
16.918 & -18.981 & 0.37 & 36.7 & 182.3 & 30.2 \\
-19.523 & -12.957 & 0.92 & 89.3 & 181.9 & 30.2 \\
-58.427 & -2.920 & 0.66 & 8.6 & 180.5 & 29.8 \\
36.181 & 44.994 & 0.83 & 57.2 & 179.8 & 29.7 \\
-27.249 & 52.149 & 0.53 & 58.0 & 174.4 & 28.5
\end{tabular}

Table 9

(Continued)

\begin{tabular}{|c|c|c|c|c|c|}
\hline $\begin{array}{l}x^{\mathrm{a}} \\
\left({ }^{\prime \prime}\right)\end{array}$ & $\begin{array}{c}y^{\mathrm{a}} \\
\left({ }^{\prime \prime}\right)\end{array}$ & $q$ & $\begin{array}{l}\Theta_{q} \\
\left(^{\circ}\right)\end{array}$ & $\left(\begin{array}{r}\sigma \\
\left(\mathrm{km} \mathrm{s}^{-1}\right)\end{array}\right.$ & $\begin{array}{r}r_{\mathrm{t}} \\
(\mathrm{kpc})\end{array}$ \\
\hline-25.792 & 57.782 & 0.75 & 56.9 & 167.0 & 26.9 \\
\hline 60.244 & -20.431 & 0.77 & 28.4 & 165.9 & 26.7 \\
\hline 65.420 & 45.366 & 0.24 & -70.9 & 159.9 & 25.4 \\
\hline-8.492 & 75.149 & 0.76 & 31.2 & 154.6 & 24.3 \\
\hline 56.269 & 39.858 & 0.67 & -88.2 & 147.2 & 22.7 \\
\hline-78.600 & -29.236 & 0.81 & 62.5 & 146.7 & 22.6 \\
\hline-5.413 & 26.620 & 0.86 & 59.3 & 144.0 & 22.1 \\
\hline 9.396 & 8.386 & 0.69 & -10.1 & 135.6 & 20.4 \\
\hline 19.473 & -3.083 & 0.80 & 25.5 & 133.9 & 20.0 \\
\hline 93.544 & -8.950 & 0.61 & -29.5 & 133.3 & 19.9 \\
\hline-8.863 & -49.294 & 0.82 & 39.6 & 133.0 & 19.9 \\
\hline 17.747 & 51.281 & 0.31 & -6.5 & 130.1 & 19.3 \\
\hline-23.885 & 17.186 & 0.84 & -8.0 & 128.2 & 18.9 \\
\hline-6.973 & -48.796 & 0.55 & -13.6 & 128.1 & 18.9 \\
\hline-1.892 & 7.503 & 0.67 & 34.0 & 124.3 & 18.2 \\
\hline 10.517 & -52.862 & 0.91 & 44.5 & 121.7 & 17.6 \\
\hline 22.649 & -53.029 & 0.94 & -7.4 & 120.6 & 17.4 \\
\hline 9.135 & -16.001 & 0.56 & 67.1 & 117.3 & 16.8 \\
\hline-8.101 & -1.448 & 0.77 & -41.9 & 116.4 & 16.6 \\
\hline 6.194 & 14.554 & 0.75 & -37.1 & 116.4 & 16.6 \\
\hline 21.592 & 5.996 & 0.79 & 18.5 & 115.7 & 16.5 \\
\hline 59.033 & 14.592 & 0.76 & 54.0 & 114.5 & 16.3 \\
\hline-29.284 & 9.803 & 0.63 & 61.5 & 111.5 & 15.7 \\
\hline-9.271 & -10.396 & 0.81 & -52.7 & 111.5 & 15.7 \\
\hline 67.743 & 5.471 & 0.48 & -81.6 & 111.2 & 15.6 \\
\hline 46.041 & -9.524 & 0.75 & 83.3 & 110.8 & 15.6 \\
\hline-13.786 & 79.453 & 0.95 & 13.8 & 109.3 & 15.3 \\
\hline-52.979 & -8.875 & 0.83 & 60.4 & 106.9 & 14.8 \\
\hline-66.483 & -38.795 & 0.87 & 38.7 & 105.6 & 14.6 \\
\hline 52.678 & -9.056 & 0.82 & -35.2 & 105.4 & 14.6 \\
\hline-9.375 & -2.275 & 0.82 & -17.6 & 105.2 & 14.5 \\
\hline-10.290 & -55.387 & 0.57 & -2.6 & 104.3 & 14.4 \\
\hline-72.341 & -21.512 & 0.71 & 13.0 & 104.0 & 14.3 \\
\hline-27.184 & 9.263 & 0.46 & 39.5 & 102.2 & 14.0 \\
\hline-1.316 & 22.373 & 0.92 & 4.4 & 102.2 & 14.0 \\
\hline 33.113 & -24.055 & 0.78 & 27.2 & 100.8 & 13.7 \\
\hline-56.983 & -13.861 & 0.79 & 19.6 & 100.5 & 13.7 \\
\hline 20.862 & -6.007 & 0.71 & -74.9 & 98.9 & 13.4 \\
\hline 77.397 & -10.026 & 0.74 & -76.1 & 97.9 & 13.2 \\
\hline-64.503 & 18.440 & 0.74 & -66.3 & 97.6 & 13.1 \\
\hline-20.902 & 16.033 & 0.53 & -34.5 & 96.0 & 12.9 \\
\hline-21.888 & -34.820 & 0.86 & 16.5 & 93.7 & 12.4 \\
\hline-29.310 & -23.851 & 0.82 & -68.7 & 92.7 & 12.3 \\
\hline 3.169 & 25.294 & 0.68 & -1.7 & 92.3 & 12.2 \\
\hline-19.579 & 16.272 & 0.44 & 77.8 & 91.7 & 12.1 \\
\hline 34.186 & -5.002 & 0.54 & -82.8 & 91.2 & 12.0 \\
\hline 84.412 & -3.159 & 0.90 & 67.9 & 89.5 & 11.7 \\
\hline-10.054 & -32.007 & 0.92 & 76.0 & 85.3 & 11.0 \\
\hline-51.558 & -28.504 & 0.63 & -35.0 & 85.1 & 10.9 \\
\hline-11.110 & -10.114 & 0.56 & 28.8 & 85.0 & 10.9 \\
\hline-56.052 & -46.963 & 0.82 & -29.5 & 84.0 & 10.8 \\
\hline-58.147 & 16.382 & 0.91 & 89.8 & 83.3 & 10.6 \\
\hline 19.309 & 18.352 & 0.54 & -55.4 & 82.6 & 10.5 \\
\hline-62.885 & 19.793 & 0.59 & 59.2 & 81.9 & 10.4 \\
\hline 44.739 & 40.229 & 0.71 & -82.0 & 78.1 & 9.8 \\
\hline-6.185 & 23.563 & 0.69 & -4.0 & 77.7 & 9.7 \\
\hline-47.318 & 20.110 & 0.42 & 5.7 & 77.3 & 9.6 \\
\hline-42.827 & -33.806 & 0.61 & 21.9 & 77.2 & 9.6 \\
\hline 18.023 & -83.677 & 0.58 & 65.9 & 76.0 & 9.4 \\
\hline 44.578 & -39.135 & 0.80 & -89.8 & 75.7 & 9.4 \\
\hline 45.326 & -39.482 & 0.76 & -40.3 & 75.7 & 9.4 \\
\hline-54.643 & 12.139 & 0.70 & -40.9 & 75.7 & 9.4 \\
\hline-68.528 & 12.943 & 0.95 & 38.1 & 75.3 & 9.3 \\
\hline-8.039 & -23.346 & 0.70 & 11.6 & 74.7 & 9.2 \\
\hline-63.008 & 9.546 & 0.18 & -37.4 & 74.0 & 9.1 \\
\hline
\end{tabular}


Table 9

(Continued)

\begin{tabular}{lrlrrr}
\hline \hline $\begin{array}{l}x^{\mathrm{a}} \\
(\prime)\end{array}$ & \multicolumn{1}{c}{$\begin{array}{c}y^{\mathrm{a}} \\
\left({ }^{\prime \prime}\right)\end{array}$} & $q$ & $\begin{array}{c}\Theta_{q} \\
\left({ }^{\circ}\right)\end{array}$ & $\begin{array}{r}\sigma \\
\left(\mathrm{km} \mathrm{s}^{-1}\right)\end{array}$ & $\begin{array}{r}r_{\mathrm{t}} \\
(\mathrm{kpc})\end{array}$ \\
\hline-20.924 & 34.030 & 0.37 & 41.5 & 71.6 & 8.7 \\
-54.231 & -35.225 & 0.82 & -35.1 & 70.5 & 8.5 \\
-25.998 & 7.123 & 0.54 & 33.7 & 68.1 & 8.1 \\
-34.878 & -43.036 & 0.49 & 71.2 & 67.7 & 8.1 \\
55.964 & -49.356 & 0.58 & -83.0 & 64.8 & 7.6 \\
28.577 & 28.456 & 0.82 & 0.9 & 64.7 & 7.6 \\
26.103 & -30.477 & 0.97 & 5.7 & 64.4 & 7.5 \\
41.714 & 41.585 & 0.67 & 40.0 & 62.5 & 7.3 \\
43.372 & -11.300 & 0.70 & -73.7 & 58.1 & 6.6 \\
20.281 & 19.628 & 0.77 & -9.3 & 57.2 & 6.5 \\
0.946 & 32.097 & 0.77 & 11.2 & 53.1 & 5.8 \\
-58.728 & 33.022 & 0.61 & 80.6 & 51.4 & 5.6 \\
44.865 & 52.272 & 0.60 & -11.5 & 45.3 & 4.7 \\
55.581 & -41.990 & 0.60 & -87.2 & 42.8 & 4.4 \\
\hline
\end{tabular}

Note. ${ }^{\text {a }}$ Relative to the center of the BCG at 12:06:12.134 R.A. (J2000), $-08: 48: 03.35$ decl. (J2000).

presented in Section 5.2. The positions are again given relative to the BCG.

\section{REFERENCES}

Andreon, S. 1996, A\&A, 314, 763

Auger, M. W., Treu, T., Bolton, A. S., et al. 2010, ApJ, 724, 511

Barbera, F. L., Lopes, P. A. A., \& de Carvalho, R. R. 2011, in The Fundamental Plane of Early-type Galaxies: Environmental Dependence from g Through

K, ed. I. Ferreras \& A. Pasquali (Heidelberg: Springer-Verlag), 79

Bender, R., Burstein, D., \& Faber, S. M. 1992, ApJ, 399, 462

Bender, R., Saglia, R. P., Ziegler, B., et al. 1998, ApJ, 493, 529

Benítez, N. 2000, ApJ, 536, 571

Benítez, N., Ford, H., Bouwens, R., et al. 2004, ApJS, 150, 1

Bernardi, M., Sheth, R. K., Annis, J., et al. 2003, AJ, 125, 1849

Bertin, E., \& Arnouts, S. 1996, A\&AS, 117, 393

Biviano, A., Rosati, P., Balestra, I., et al. 2013, A\&A, submitted (arXiv:1307.5867)

Bolton, A. S., Treu, T., Koopmans, L. V. E., et al. 2008, ApJ, 684, 248

Brainerd, T. G., Blandford, R. D., \& Smail, I. 1996, ApJ, 466, 623

Brimioulle, F., Seitz, S., Lerchster, M., Bender, R., \& Snigula, J. 2013, MNRAS, 432, 1046

Broadhurst, T., Benítez, N., Coe, D., et al. 2005, ApJ, 621, 53

Budzynski, J. M., Koposov, S. E., McCarthy, I. G., McGee, S. L., \& Belokurov, V. 2012, MNRAS, 423, 104

Coe, D., Benítez, N., Broadhurst, T., \& Moustakas, L. A. 2010, ApJ, 723, 1678

Coe, D., Benítez, N., Sánchez, S. F., et al. 2006, AJ, 132, 926

Colley, W. N., Tyson, J. A., \& Turner, E. L. 1996, ApJL, 461, L83

de Vaucouleurs, G. 1948, AnAp, 11, 247

Diego, J. M., Sandvik, H. B., Protopapas, P., et al. 2005, MNRAS, 362, 1247

Diemand, J., Kuhlen, M., \& Madau, P. 2007, ApJ, 667, 859

Donnarumma, A., Ettori, S., Meneghetti, M., et al. 2011, A\&A, 528, A73

Dressler, A. 1980, ApJ, 236, 351

Dressler, A., Oemler, A., Jr., Couch, W. J., et al. 1997, ApJ, 490, 577

Dunkley, J., Bucher, M., Ferreira, P. G., Moodley, K., \& Skordis, C. 2005, MNRAS, 356, 925

Ebeling, H., Ma, C. J., Kneib, J.-P., et al. 2009, MNRAS, 395, 1213

Eichner, T., Seitz, S., \& Bauer, A. 2012, MNRAS, 427, 1918

Elíasdóttir, Á., Limousin, M., Richard, J., et al. 2007, arXiv:0710.5636

Faber, S. M., \& Jackson, R. E. 1976, ApJ, 204, 668

Focardi, P., \& Malavasi, N. 2012, ApJ, 756, 117

Fritz, A., Böhm, A., \& Ziegler, B. L. 2009, MNRAS, 393, 1467

Gao, L., De Lucia, G., White, S. D. M., \& Jenkins, A. 2004a, MNRAS, $352, \mathrm{~L} 1$

Gao, L., White, S. D. M., Jenkins, A., Stoehr, F., \& Springel, V. 2004b, MNRAS, 355,819
Gavazzi, R., Treu, T., Rhodes, J. D., et al. 2007, ApJ, 667, 176

Geiger, B., \& Schneider, P. 1999, MNRAS, 302, 118

Gerhard, O., Kronawitter, A., Saglia, R. P., \& Bender, R. 2001, AJ, 121, 1936

Ghigna, S., Moore, B., Governato, F., et al. 1998, MNRAS, 300, 146

Golse, G., \& Kneib, J.-P. 2002, A\&A, 390, 821

Grillo, C., Eichner, T., Seitz, S., et al. 2010, ApJ, 710, 372

Grillo, C., Gobat, R., Lombardi, M., \& Rosati, P. 2009, A\&A, 501, 461

Halkola, A., Hildebrandt, H., Schrabback, T., et al. 2008, A\&A, 481, 65

Halkola, A., Seitz, S., \& Pannella, M. 2006, MNRAS, 372, 1425

Halkola, A., Seitz, S., \& Pannella, M. 2007, ApJ, 656, 739

Hoekstra, H., Franx, M., Kuijken, K., Carlberg, R. G., \& Yee, H. K. C. 2003, MNRAS, 340, 609

Hoekstra, H., Yee, H. K. C., \& Gladders, M. D. 2004, ApJ, 606, 67

Host, O. 2012, MNRAS, 420, L18

Jullo, E., Natarajan, P., Kneib, J.-P., et al. 2010, Sci, 329, 924

Knebe, A., Yahagi, H., Kase, H., Lewis, G., \& Gibson, B. K. 2008, MNRAS, 388, L34

Komatsu, E., Smith, K. M., Dunkley, J., et al. 2011, ApJS, 192, 18

Koopmans, L. V. E., Treu, T., Bolton, A. S., Burles, S., \& Moustakas, L. A. 2006, ApJ, 649, 599

Kormendy, J., \& Bender, R. 2013, ApJL, 769, L5

Limousin, M., Kneib, J. P., Bardeau, S., et al. 2007a, A\&A, 461, 881

Limousin, M., Richard, J., Jullo, E., et al. 2007b, ApJ, 668, 643

Limousin, M., Richard, J., Kneib, J.-P., et al. 2008, A\&A, 489, 23

Limousin, M., Sommer-Larsen, J., Natarajan, P., \& Milvang-Jensen, B. 2009, ApJ, 696, 1771

Mandelbaum, R., Seljak, U., Cool, R. J., et al. 2006, MNRAS, 372, 758

Matković, A., \& Guzmán, R. 2005, MNRAS, 362, 289

Merritt, D. 1983, ApJ, 264, 24

Merritt, D. 1984, ApJ, 276, 26

Narayan, R. 1998, NewAR, 42, 73

Natarajan, P., Kneib, J.-P., \& Smail, I. 2002a, ApJL, 580, L11

Natarajan, P., Loeb, A., Kneib, J.-P., \& Smail, I. 2002b, ApJL, 580, L17

Navarro, J. F., Frenk, C. S., \& White, S. D. M. 1997, ApJ, 490, 493

Nelson, E. J., van Dokkum, P. G., Brammer, G., et al. 2012, ApJL, 747, L28

Nigoche-Netro, A., Ruelas, A., \& Sánchez, L. J. 2011, RMxAA, 40, 126

Peng, C. Y., Ho, L. C., Impey, C. D., \& Rix, H.-W. 2010, AJ, 139, 2097

Postman, M., Coe, D., Benítez, N., et al. 2012, ApJS, 199, 25

Pu, S. B., Saglia, R. P., Fabricius, M. H., et al. 2010, A\&A, 516, A4

Richard, J., Kneib, J.-P., Limousin, M., Edge, A., \& Jullo, E. 2010a, MNRAS, 402, L44

Richard, J., Smith, G. P., Kneib, J.-P., et al. 2010b, MNRAS, 404, 325

Romanishin, W. 1986, AJ, 91, 76

Rusin, D., Kochanek, C. S., Falco, E. E., et al. 2003, ApJ, 587, 143

Saglia, R. P., Sánchez-Blázquez, P., Bender, R., et al. 2010, A\&A, 524, A6

Sand, D. J., Treu, T., Smith, G. P., \& Ellis, R. S. 2004, ApJ, 604, 88

Schneider, P., \& Rix, H.-W. 1997, ApJ, 474, 25

Seitz, S., Saglia, R. P., Bender, R., et al. 1998, MNRAS, 298, 945

Sérsic, J. L. 1963, BAAA, 6, 41

Suyu, S. H., \& Halkola, A. 2010, A\&A, 524, A94

Suyu, S. H., Hensel, S. W., McKean, J. P., et al. 2012, ApJ, 750, 10

Suyu, S. H., Marshall, P. J., Hobson, M. P., \& Blandford, R. D. 2006, MNRAS, 371, 983

Thomas, D., Maraston, C., Bender, R., \& Mendes de Oliveira, C. 2005, ApJ, 621,673

Thomas, J., Saglia, R. P., Bender, R., et al. 2009, ApJ, 691, 770

Umetsu, K., Medezinski, E., Nonino, M., et al. 2012, ApJ, 755, 56

van Dokkum, P. G., Brammer, G., Fumagalli, M., et al. 2011, ApJL, 743, L15

van Dokkum, P. G., \& van der Marel, R. P. 2007, ApJ, 655, 30

Ventimiglia, G., Arnaboldi, M., \& Gerhard, O. 2011, A\&A, 528, A24

Warnick, K., Knebe, A., \& Power, C. 2008, MNRAS, 385, 1859

Warren, S. J., \& Dye, S. 2003, ApJ, 590, 673

Wegner, G. A., Corsini, E. M., Thomas, J., et al. 2012, AJ, 144, 78

Wilman, D. J., \& Erwin, P. 2012, ApJ, 746, 160

Wright, C. O., \& Brainerd, T. G. 2000, ApJ, 534, 34

Wuyts, S., Förster Schreiber, N. M., Genzel, R., et al. 2012, ApJ, 753, 114

Ziegler, B. L., \& Bender, R. 1997, MNRAS, 291, 527

Zitrin, A., Bartelmann, M., Umetsu, K., Oguri, M., \& Broadhurst, T. 2012a, MNRAS, 426, 2944

Zitrin, A., Broadhurst, T., Barkana, R., Rephaeli, Y., \& Benítez, N. 2011, MNRAS, 410, 1939

Zitrin, A., Meneghetti, M., Umetsu, K., et al. 2013, ApJL, 762, L30

Zitrin, A., Rosati, P., Nonino, M., et al. 2012b, ApJ, 749, 97 